\title{
Intestinal proinflammatory macrophages induce a phenotypic switch in interstitial cells of Cajal
}

\author{
Xuyong Chen, ${ }^{1}$ Xinyao Meng, ${ }^{1}$ Hongyi Zhang, ${ }^{1}$ Chenzhao Feng, ${ }^{2}$ Bin Wang, ${ }^{3}$ Ning Li, ${ }^{1}$ Khalid Mohamoud Abdullahi, ${ }^{1}$ Xiaojuan Wu, \\ Jixin Yang, ${ }^{1}$ Zhi Li, ${ }^{1}$ Chunlei Jiao, ${ }^{1}$ Jia Wei, ${ }^{1}$ Xiaofeng Xiong, ${ }^{4}$ Kang Fu, ${ }^{4}$ Lei Yu, ${ }^{4}$ Gail E. Besner, ${ }^{5}$ and Jiexiong Feng ${ }^{1}$ \\ 'Department of Pediatric Surgery, Tongji Hospital, Tongji Medical College; and ' 2 Tongji Medical College, Huazhong University of Science and Technology, Wuhan, China. ${ }^{3}$ Department of Pediatric Surgery, \\ Shenzhen Children's Hospital, Shenzhen, China. ${ }^{4}$ Department of Neonatal Surgery, Wuhan Children's Hospital, Tongji Medical College, Huazhong University of Science and Technology, Wuhan, China. \\ ${ }^{5}$ Department of Pediatric Surgery, Center for Perinatal Research, Nationwide Children's Hospital, The Ohio State University, Columbus, Ohio, USA.
}

\begin{abstract}
Interstitial cells of Cajal (ICCs) are pacemaker cells in the intestine, and their function can be compromised by loss of C-KIT expression. Macrophage activation has been identified in intestine affected by Hirschsprung diseaseassociated enterocolitis (HAEC). In this study, we examined proinflammatory macrophage activation and explored the mechanisms by which it downregulates C-KIT expression in ICCs in colon affected by HAEC. We found that macrophage activation and TNF- $\alpha$ production were dramatically increased in the proximal dilated colon of HAEC patients and 3-week-old Ednrb-/- mice. Moreover, ICCs lost their C-KIT+ phenotype in the dilated colon, resulting in damaged pacemaker function and intestinal dysmotility. However, macrophage depletion or TNF- $\alpha$ neutralization led to recovery of ICC phenotype and restored their pacemaker function. In isolated ICCs, TNF- $\alpha$-mediated phosphorylation of p65 induced overexpression of microRNA-221 (miR-221), resulting in suppression of C-KIT expression and pacemaker currents. We also identified a TNF- $\alpha / N F-\kappa B / m i R-221$ pathway that downregulated C-KIT expression in ICCs in the colon affected by HAEC. These findings suggest the important roles of proinflammatory macrophage activation in a phenotypic switch of ICCs, representing a promising therapeutic target for HAEC.
\end{abstract}

\section{Introduction}

Hirschsprung disease (HSCR) is a birth defect characterized by the absence of ganglion cells in the distal bowel (1). HSCR-associated enterocolitis (HAEC), the most common and serious complication of HSCR, is a life-threatening condition that can occur from the neonatal period through adolescence. The morbidity and mortality of HAEC remain unchanged even after a properly performed pull-through operation (2). The pathogenesis of HAEC is unclear, although a breached intestinal barrier and abnormal immunity have been reported to be important in the development of HAEC (3).

Interstitial cells of Cajal (ICCs) are pacemaker cells responsible for generating slow waves in the gastrointestinal tract (4). Not surprisingly, reduced C-KIT ${ }^{+}$ICCs are noted in the aganglionic bowel and the transition zone of HSCR patients (5-7). Reduced myenteric ICCs have also been found in the proximal ganglionic bowel (8). The loss of C-KIT expression, whose signaling maintains the pacemaker function of ICCs, promotes a phenotypic switch of C-KIT ${ }^{+}$ICCs into a C-KIT' phenotype (9, 10). The mechanisms of this ICC phenotypic switch in HSCR patients are unclear. We previously reported that LPS-induced

Conflict of interest: The authors have declared that no conflict of interest exists. Copyright: () 2020, American Society for Clinical Investigation.

Submitted: December 10, 2018; Accepted: August 13, 2020; Published: November 3, 2020. Reference information: / Clin Invest. 2020;130(12):6443-6456.

https://doi.org/10.1172/JCl126584. inflammation inhibited the expression of C-KIT in murine colon (11). Therefore, it is speculated that the loss of C-KIT ${ }^{+}$phenotype in ICCs is due to the presence of proinflammatory factors in the colon of HSCR patients.

As an important part of first-line defense mechanisms, intestinal macrophages protect the mucosa against harmful pathogens, act as effector cells, and scavenge dead cells and debris (12). We and others have found that in inflamed intestinal mucosa, classically activated macrophages (M1) infiltrate the intestine to induce inflammation, which plays a key role in several diseases, including inflammatory bowel disease and necrotizing enterocolitis (13, 14). There is increasing evidence supporting the roles of macrophages in regulating gastrointestinal motility in inflammatory diseases. Previous studies found that LPS induced the expression of inducible nitric oxide synthase (iNOS) in resident macrophages, resulting in nitric oxide-induced phenotypic switches in ICCs (15). Moreover, tumor necrosis factor- $\alpha$ (TNF- $\alpha$ ) secreted from M1 macrophages resulted in direct injury to ICCs (16).

To confirm the role of macrophages in the phenotypic switches of ICCs in the colon affected by HAEC, we examined specimens from humans as well as endothelin receptor B (Ednrb) gene-knockout mice with HSCR. To explore the mechanisms by which macrophages damage ICC networks, we investigated macrophage polarization and the phenotypic switch of ICCs during the development of HAEC. We have also identified a potential mechanism by which proinflammatory macrophages inhibited C-KIT expression in ICCs via the TNF- $\alpha$ / NF- $\mathrm{B} /$ microRNA-221 (miR-221) pathway. 
A

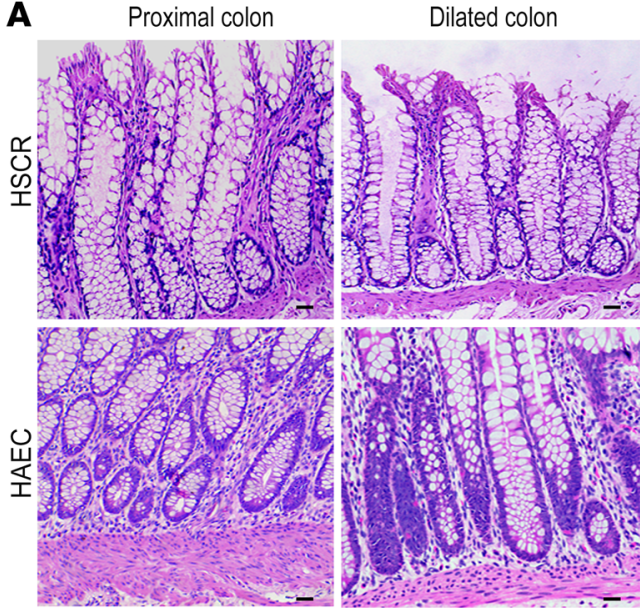

Transition zone
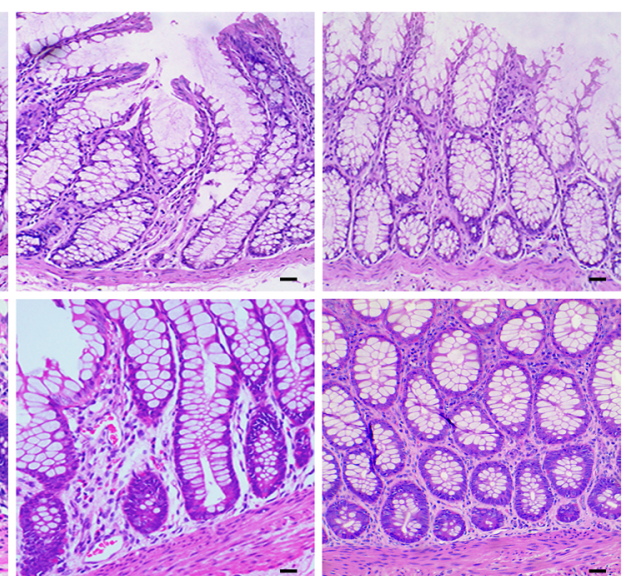

B

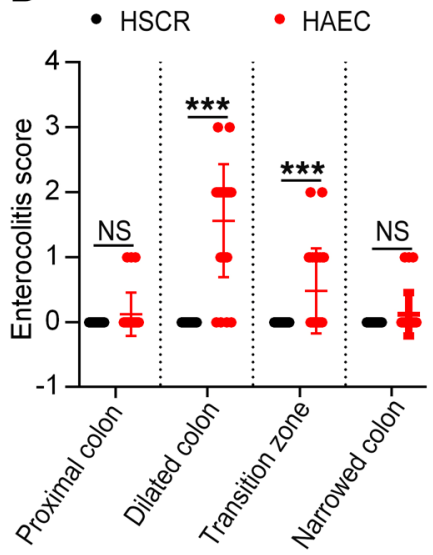

C

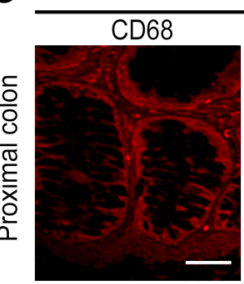

HSCR
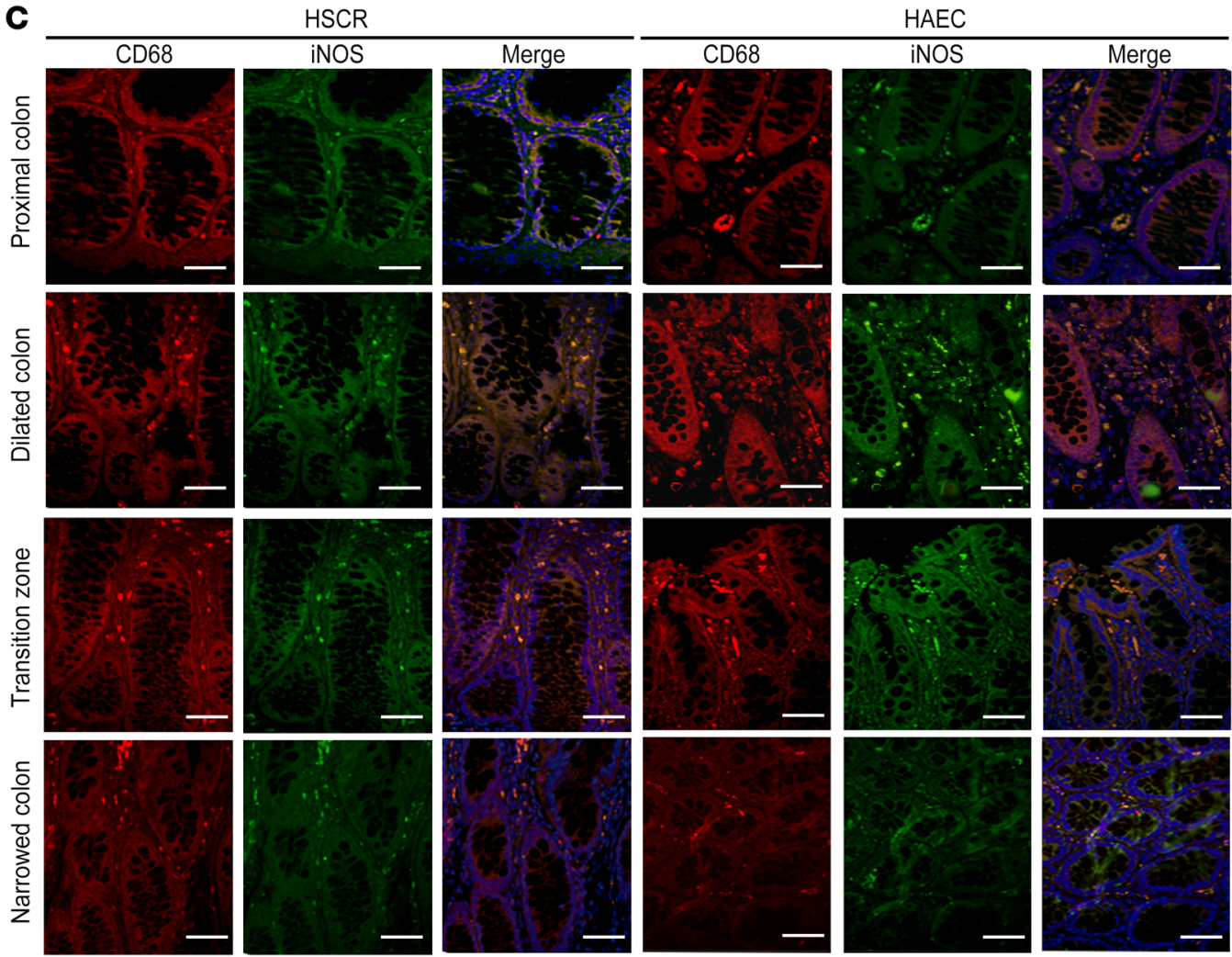

Figure 1. Enterocolitis score increases and macrophages infiltrate in the dilated colon of HAEC patients. (A) H\&E staining of the proximal resection margin, dilated segment, transition zone, and narrowed colon of HSCR and HAEC patients. Scale bars: $100 \mu \mathrm{m}$. (B) An enterocolitis grading system was used to evaluate inflammation. (C) CD68 and iNOS immunofluorescence double staining to identify M1 macrophages. Red, CD68; green, iNOS; blue, DAPI. Scale bars: $100 \mu \mathrm{m}$. Data shown are representative of $n=75$ samples of HSCR patients and $n=21$ samples of HAEC patients. One-way ANOVA: ${ }^{* *} P<0.001$.

\section{Results}

Proinflammatory M1 macrophages infiltrate the dilated colon affected by enterocolitis in HSCR patients. A total of 96 patients, who underwent pull-through procedures at our institution and were pathologically diagnosed with HSCR, were recruited in this study. HAEC was diagnosed according to established diagnostic criteria (17). Twenty-one patients diagnosed with HAEC were enrolled in the HAEC group. The remaining 75 patients were enrolled in the HSCR group.
Of the 21 HAEC patients, multiple crypt abscesses and immune cell infiltration were found in the dilated colon. A few crypt abscesses were also found in the transition zone. No crypt abscess was present in the distal narrow colon or the normal proximal colon (Figure 1A). Consistent with crypt abscess and immune cell distribution, enterocolitis scores were highest in the dilated colon, followed by the transition zone, the distal colon, and the proximal normal colon (Figure 1B).

Of the 75 HSCR patients, no crypt abscess or immune cell infiltration was found in any of the 4 segments of the colon. 
A

Proximal colon

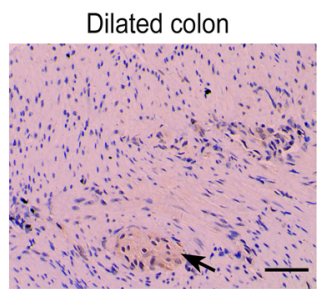

Transition zone

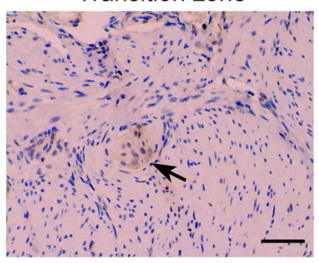

Narrowed colon
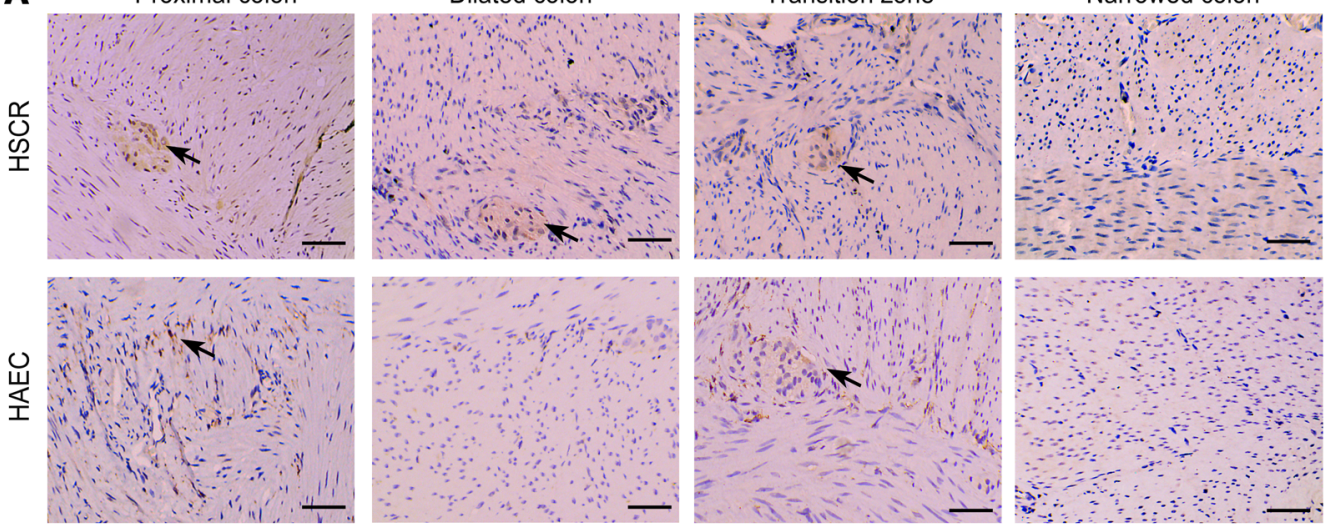

B

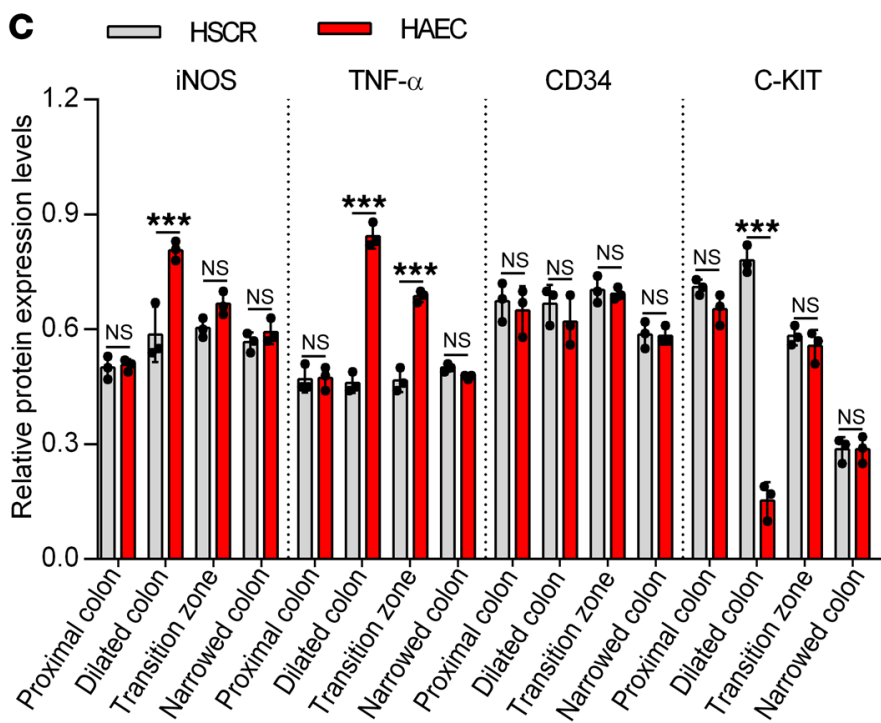

Figure 2. Proinflammatory cytokines increase, whereas C-KIT+ ICCs decrease, in the dilated colon of HAEC patients. (A) Immunohistochemistry staining of ICCs of HSCR and HAEC patients for C-KIT. Scale bars: $100 \mu \mathrm{m}$. Data are representative of $n=75$ samples of HSCR patients and $n=21$ samples of HAEC patients. Arrows indicate C-KIT+ ICCs. (B) Western blotting of colon of HSCR and HAEC patients. (C) Semiquantitative analysis of protein expression levels, with each protein being normalized to $\beta$-actin. One-way ANOVA: ${ }^{* *} P<0.001$.

Enterocolitis was scored as $\mathrm{O}$ in the 4 colon segments in these patients (Figure $1, \mathrm{~A}$ and $\mathrm{B}$ ).

CD68 and iNOS were used to identify proinflammatory M1 macrophages in tissue sections (13). Significantly increased M1 macrophages were found in the dilated colon of HAEC patients, accompanied by slightly increased M1 macrophages in the transition zone (Figure 1C). However, M1 macrophages were barely detectable in any of the 4 colon segments of HSCR patients (Figure 1C). In the HAEC group, compared with the other 3 segments, the dilated colon expressed the highest levels of iNOS and TNF- $\alpha$ as detected by Western blotting (Figure 2, $\mathrm{B}$ and $\mathrm{C}$ ) and real-time PCR (Supplemental Figure 1A; supplemental material available online with this article; https://doi. org/10.1172/JCI126584DS1).

These observations demonstrate prominent infiltration of proinflammatory M1 macrophages and increased expression of iNOS and TNF- $\alpha$ in the dilated colon of HAEC patients.

ICCs lose their C-KIT $T^{+}$phenotype in the dilated colon of $H A E C$ patients. ICCs are the only intestinal cells that express both C-KIT and CD34 (11). To study the phenotypic switches of ICCs, we identified the distribution of C-KIT ${ }^{+}$ICCs in the colon by immunohistochemistry (IHC) staining. No C-KIT ${ }^{+}$ ICCs were found in the distal narrow colon of either the HAEC or the HSCR group. Compared with the HSCR group, C-KIT ${ }^{+}$ ICCs were significantly reduced in the dilated colon of HAEC patients. Moreover, ICCs were normally distributed in the proximal normally ganglionated colon of HAEC and HSCR patients (Figure 2A).

The protein and mRNA levels of C-KIT and CD34 were examined by Western blotting and real-time PCR, respectively. We found that C-KIT expression was lowest in the dilated colon of the HAEC group (Figure 2, B and C; and Supplemental Figure 1B). No differences of CD34 expression were found between the HAEC and HSCR groups (Figure 2, B and C; and Supplemental Figure 1B). These results show a loss of C-KIT expression in ICCs in the proximal dilated colon affected by HAEC.

ICCs lose their C-KIT $T^{+}$phenotype in the proximal dilated colon of Ednrb ${ }^{-/-}$mice. We used Ednrb-null mice as a well-established 

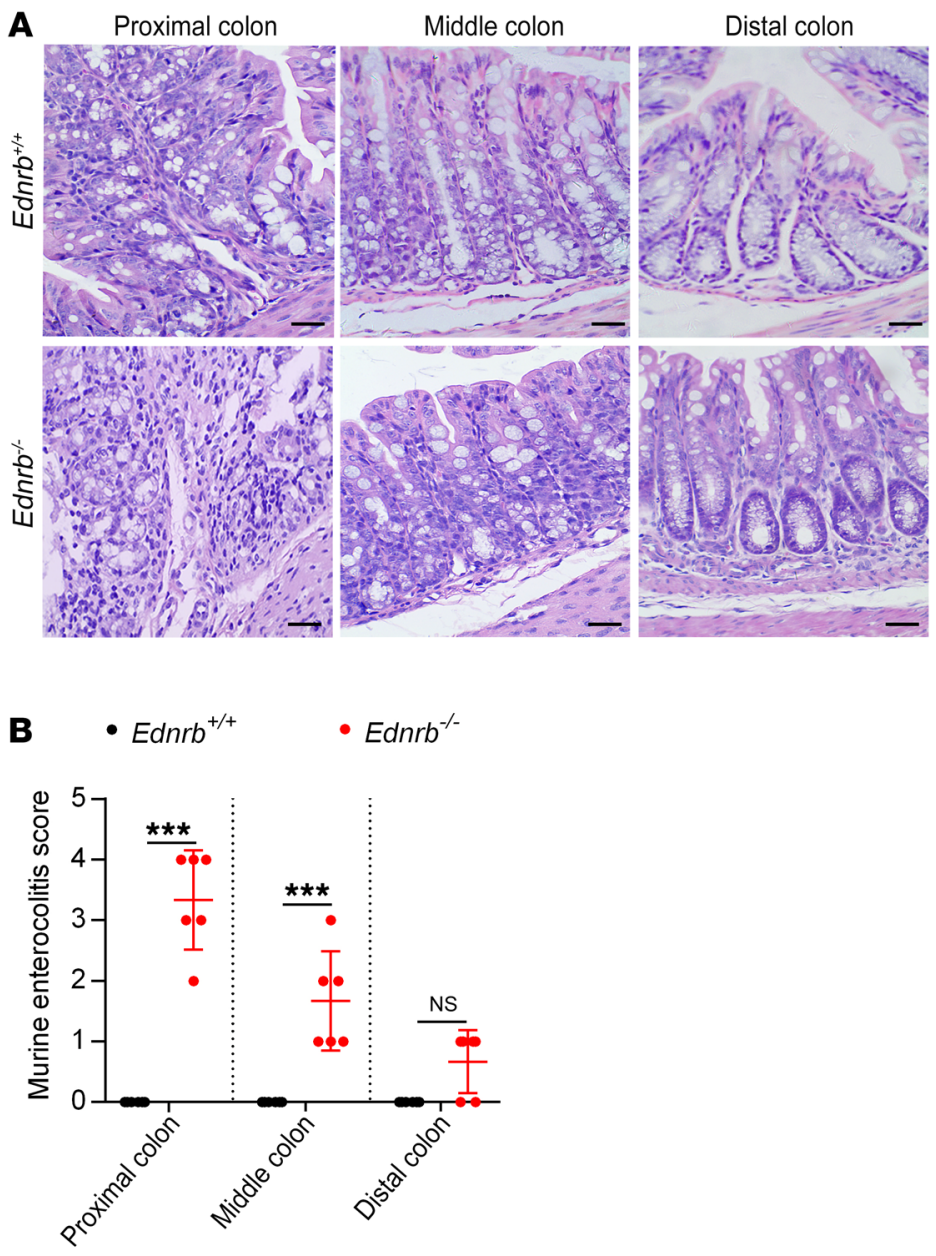

Figure 3. Enterocolitis score increases in the proximal colon of 3-weekold Edn rb $\boldsymbol{b}^{-/-}$mice. (A) H\&E staining of proximal, middle, and distal colon sections from 3-week-old Ednrb ${ }^{+/+}$and $E_{d n r b^{-/-}}$mice. Scale bars: $100 \mu \mathrm{m}$. (B) An enterocolitis grading system was used to evaluate inflammation. Data shown represent results from 6 independent experiments. One-way ANOVA: ${ }^{* *} P<0.001$.

model for HSCR (18). The majority of Ednrb ${ }^{-/-}$mice developed enterocolitis by the third week of life and died in the following $1-2$ weeks (19).

There were no inflammatory cells in the colon of 1- or 2-weekold $E d n r^{-/}$mice (Supplemental Figure 2, A-D). However, at the third week of life, inflammatory cells and enterocolitis scores were dramatically increased in the proximal dilated colon (Figure 3, A and B), indicating the development of HAEC.

Ganglion cells were detected by double staining of NeuN and PGP9.5, which are specific makers of enteric neurons $(20,21)$. We found that NeuN ${ }^{+}$PGP9. $5^{+}$cells were absent in the distal colon, whereas NeuN ${ }^{+}$PGP9. $5^{+}$enteric neurons were present in the middle and proximal colon of 1-, 2-, or 3-week-old Ednrb ${ }^{-/}$mice (Supplemental Figure 3).

The C-KIT ligand stem cell factor (SCF) in the intestine is mostly secreted by enteric neurons (22). Of the 1-, 2-, and 3-weekold $E d n r b^{-/}$mice, SCF expression was decreased in the distal colon but was normally expressed in the middle and proximal colon as detected by Western blotting (Supplemental Figure 4, A-D; and
Figure 4, B and C) and real-time PCR (Supplemental Figure 5, A and B; and Supplemental Figure 6B), consistent with neuronal cell distribution.

Regardless of the presence of HAEC, there were no C-KIT ${ }^{+}$ICCs in the distal colon of 1-, 2-, and 3-week-old Edn$r b^{-/}$mice. C-KIT ${ }^{+}$ICCs were normally distributed in the middle and proximal colon of 1- and 2-week-old Ednrb ${ }^{-/-}$mice but were significantly decreased in middle and proximal dilated colon of 3-week-old Ednrb-/ mice (Supplemental Figure 7, A and B; and Figure 4A). The expression of C-KIT in the colon was also assessed by Western blotting (Supplemental Figure 4, A-D; and Figure 4, B and C) and real-time PCR (Supplemental Figure 5, A and B; and Supplemental Figure 6B), which was consistent with the distribution pattern of C-KIT ${ }^{+}$ ICCs detected by IHC.

There were no significant differences in CD34 expression between $E d n r b^{-/-}$mice and $E d n r b^{+/+}$mice (wild type [WT]) regardless of age or colon segment (Supplemental Figure 4, A-D; Figure 4, B and C; Supplemental Figure 5, A and B; and Supplemental Figure 6B). Moreover, we studied another specific marker of ICCs, ANO1 expression (23). We found that ANO1 immunoreactivity was colocalized with C-KIT immunoreactivity in ICCs. The decrease in $\mathrm{ANO}^{+}$ ICCs was similar to the decrease in C-KIT ${ }^{+}$ICCs in colon tissue affected by HAEC. Furthermore, the numbers of ICCs identified in the colon by ANO1 and C-KIT immunoreactivity were nearly identical (Supplemental Figure 8, A and B; and Supplemental Figure 9).

The parallel loss of both C-KIT and ANO1, but unchanged CD34 expression, further manifests a phenotypic switch of the ICCs in proximal colon affected by HAEC.

Proinflammatory M1 macrophages infiltrate the proximal

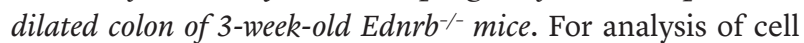
types, cells double-positive for $\mathrm{CD} 45$ and F4/80 were gated as monocytes. $\mathrm{F} 4 / 8 \mathrm{O}^{+} \mathrm{CD} 11 \mathrm{~b}^{+} \mathrm{CD} 11 \mathrm{c}^{-}$is commonly used as a marker of intestinal macrophages. Intestinal macrophages that express iNOS and TNF- $\alpha$ were defined as proinflammatory macrophages $(24,25)$.

In the distal, middle, and proximal colon of 1- and 2-weekold $E_{n n r b^{--}}$mice, the percentages of $\mathrm{CD} 45^{+} \mathrm{F} 4 / 80^{+}$monocytes

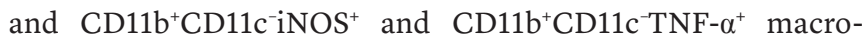
phages were not significantly different between WT and $E d n r^{-1-}$ mice (Supplemental Figures 10 and 11). However, in 3-week-old $\mathrm{Ednrb}^{-/-}$mice, $\mathrm{CD} 45^{+} \mathrm{F} 4 / 80^{+}$monocytes were increased in the proximal dilated and middle colon (Figure 5, A and B). Moreover, the percentages of $\mathrm{CD}_{11} \mathrm{~b}^{+} \mathrm{CD} 11 \mathrm{c}^{-} \mathrm{TNF}-\alpha^{+}$and $\mathrm{CD} 11 \mathrm{~b}^{+} \mathrm{CD} 11 \mathrm{c}^{-}$ iNOS $^{+}$macrophages were significantly increased from the distal to the proximal colon of $\mathrm{Ednrb}^{-/-}$mice (Figure 5, A, C, and D). Overlapping staining of CD 68 and TNF- $\alpha$ confirmed that TNF- $\alpha$ was derived from macrophages in 3-week-old Ednrb ${ }^{-/}$mice (Supplemental Figure 12). These observations confirm that proinflammatory M1 macrophages infiltrate into the proximal colon affected by HAEC.

There were no differences in TNF- $\alpha$ and iNOS expression between 1- and 2-week-old Ednrb ${ }^{-/}$mice by Western blotting (Supplemental Figure 4, A-D) or real-time PCR (Supplemental Figure 5, A and B). However, in 3-week-old Ednrb-/ mice, TNF- $\alpha$ 
A

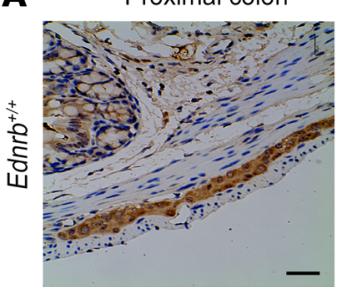

Middle colon
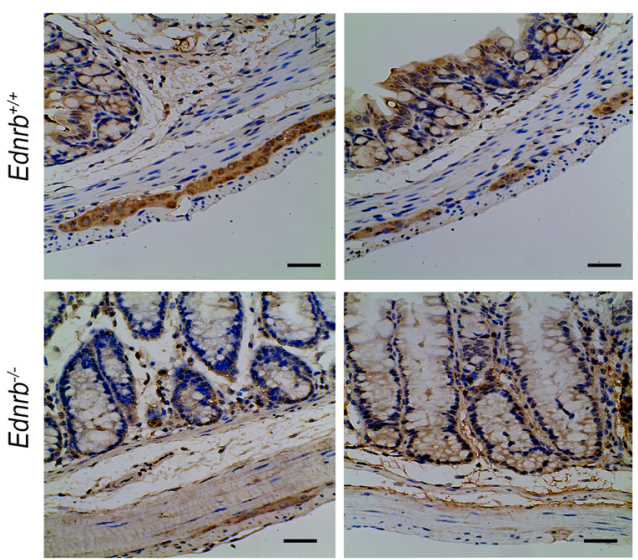

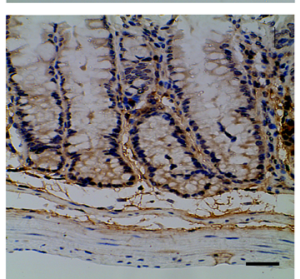

Distal colon
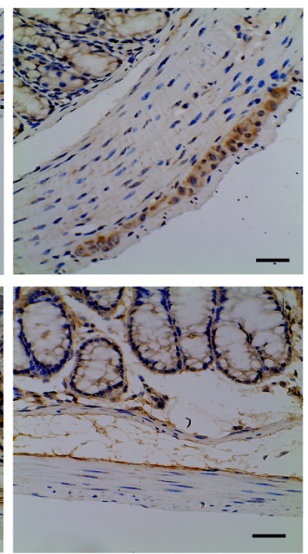

B

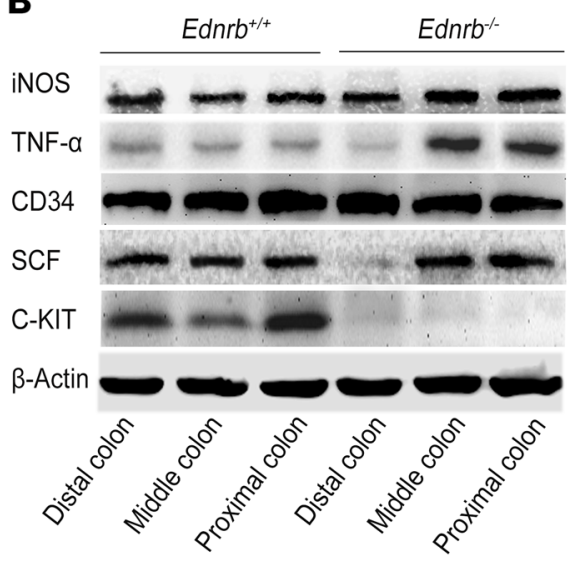

C

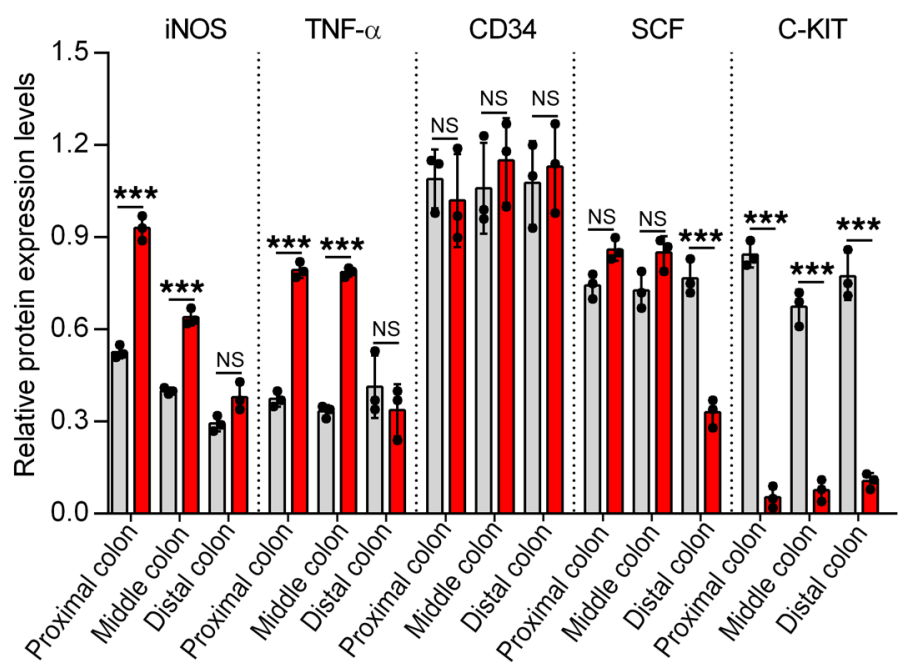

Figure 4. Proinflammatory cytokines increase but C-KIT+ ICCs decrease in the proximal colon of 3-week-old Ednrb-/- mice. (A) Immunohistochemistry staining of ICCs from 3-week-old $E_{n n r b^{+/+}}$and Ednrb ${ }^{-/-}$mice for C-KIT. Scale bars: $100 \mu \mathrm{m}$. (B) Western blotting of colon from 3-week-old $\mathrm{Ednrb}^{+/+}$and $\mathrm{Edn}$ $r b^{-/-}$mice. (C) Semiquantitative analysis of protein expression levels, with each protein being normalized to $\beta$-actin. Data shown represent results from 3 independent experiments. One-way ANOVA: ${ }^{* *} P<0.001$ and iNOS were dramatically increased in the proximal colon (Figure 4, B and C; and Supplemental Figure 6A). These observations indicate that proinflammatory M1 macrophages and cytokines increased over time in the middle and proximal colon during the first 3 weeks of life in $E d n r b^{-/-}$mice.

These observations show that HAEC mostly occurs in the proximal colon of $\mathrm{Ednrb}^{-/}$mice at the third week after birth, accompanied by increased $\mathrm{CD} 45^{+} \mathrm{F} 4 / 80^{+} \mathrm{CD} 11 \mathrm{~b}^{+} \mathrm{CD} 11 \mathrm{c}^{-}$macrophage infiltration and TNF- $\alpha$ /iNOS levels, with the loss of the $\mathrm{C}^{-\mathrm{KIT}^{+}}$phenotype in ICCs. These findings suggest a correlation between the loss of ICC phenotype and $\mathrm{CD} 45^{+} \mathrm{F} 4 / 80^{+} \mathrm{CD} 11 \mathrm{~b}^{+} \mathrm{C}-$ D11c $c^{-}$proinflammatory M1 macrophage polarization.

Depletion of macrophages restores ICC phenotype and colonic slow waves in Ednrb-/ mice. Macrophages were depleted by i.p. injection of liposomal clodronate (Clod) (26) in 2-week-old $E d n r b^{-/-}$and $E d n r b^{+/+}$mice, and the mice were sacrificed 1 week later. In 3-week-old Ednrb ${ }^{-/-}$mice, Clod treatment significantly reduced enterocolitis scores (Figure 6, A and B), decreased $\mathrm{CD} 45^{+} \mathrm{F} 4 / 80^{+}$monocytes (Figure $7, \mathrm{~A}$ and $\mathrm{B}$ ), diminished $\mathrm{CD} 11 \mathrm{~b}^{+} \mathrm{CD} 11 \mathrm{c}^{-}$macrophages, and decreased the subtypes of TNF- $\alpha^{+}$and iNOS $^{+}$cells (Figure 7, A, C, and D; Figure 8, B and C; and Supplemental Figure 13A). These results indicate that Clod reduces the activation and polarization of $\mathrm{CD} 45^{+} \mathrm{F} 4 / 80^{+} \mathrm{CD}$ $11 \mathrm{~b}^{+} \mathrm{CD} 11 \mathrm{c}^{-\mathrm{TNF}}-\alpha^{+}$proinflammatory M1 macrophages.

IHC staining demonstrated that Clod treatment increased the number of C-KIT ${ }^{+}$ICCs in the proximal and middle colon of 3-week-old Ednrb-/- mice (Figure 8A). C-KIT expression was significantly increased in the proximal dilated colon of 3-week-old $\mathrm{Ednrb}^{-/-}$mice treated with Clod, as confirmed by Western blotting (Figure 8, B and C) and real-time PCR (Supplemental Figure 13B). All colon segments expressed the same levels of CD34 protein in $\mathrm{Ednrb}^{-/-}$and WT mice (Figure 8, B and C; and Supplemental Figure 13B). Since ICCs are responsible for the generation and propagation of slow waves in the intestine (27), we next examined colonic slow waves in $\mathrm{Ednrb}^{-/-}$mice (Figure 9A). In 1- and 2-week-old Edn$\mathrm{rb}^{-/}$mice, reduced amplitudes but unaffected frequencies of slow waves were found (Figure 9, B and C). However, in 3-week-old Edn$r b^{-/-}$mice, both the frequency and the amplitude of the colonic slow waves were reduced (Figure 9, B and C). Clod treatment increased the frequency of colonic slow waves (Figure 9C).

These findings suggest that depletion of macrophages by Clod eliminates $\mathrm{CD}^{2} 5^{+} \mathrm{F} 4 / 80^{+} \mathrm{CD} 11 \mathrm{~b}^{+} \mathrm{CD} 11 \mathrm{c}^{-} \mathrm{TNF}-\alpha^{+}$proinflammatory M1 macrophages and restores C-KIT ${ }^{+}$ICCs phenotype and rhythmic colonic slow waves in mice with HAEC. 
A

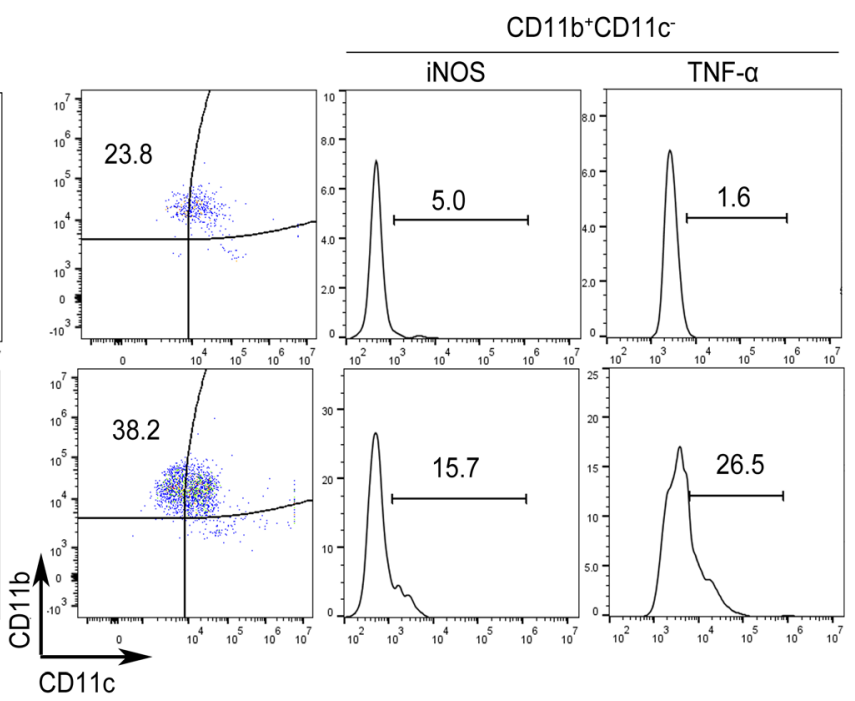

B

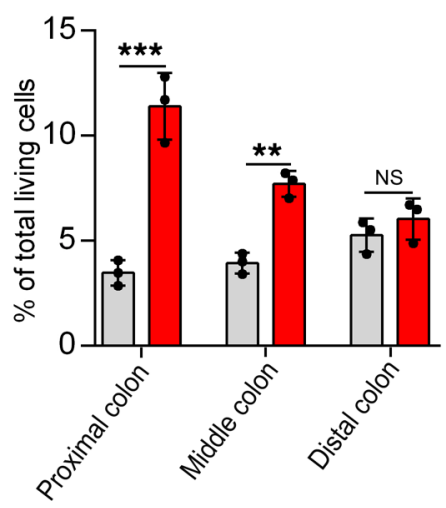

C

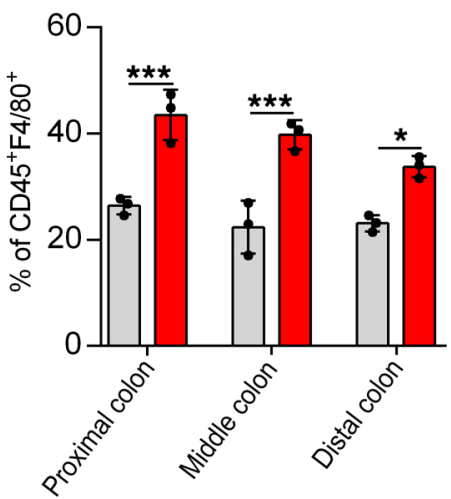

D iNOS

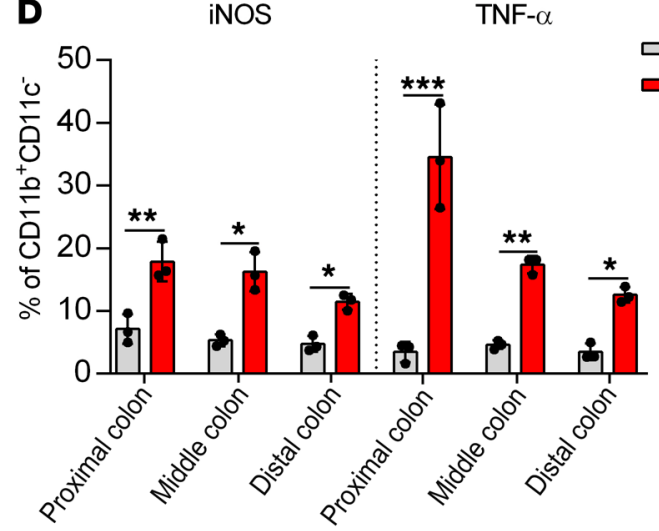

Figure 5. Proinflammatory M1 macrophages increase in the proximal colon of 3-week-old Ednrb-/- mice. (A) Representative analysis of $\mathrm{CD}_{45} \mathrm{~F}^{+} / 80^{+}, \mathrm{CD} 11 \mathrm{~b}^{+} \mathrm{CD}_{11 \mathrm{c}^{-}}$, iNOS ${ }^{+}$, and TNF- $\alpha^{+}$cells in the proximal colon from 3-week-old Ednrb $\mathrm{b}^{+/+}$and Ednrb ${ }^{-/-}$mice. (B) Percentage of $\mathrm{CD} 45^{+} \mathrm{F} 4 / 80^{+}$cells among total viable cells in colonic tissue. (C) Percentage of CD11b+CD11c cells among CD45 $\mathrm{b}^{+} \mathrm{F} / 80^{+}$cells. (D) Percentage of iNOS+ cells and TNF- $\alpha^{+}$cells among CD11 $\mathrm{b}^{+}$CD11c ${ }^{-}$cells. Data shown represent results from 3 independent experiments. One-way ANOVA: ${ }^{*} P<0.05$; ${ }^{* *} P<0.01 ;{ }^{* *} P<0.001$.

TNF- $\alpha$ neutralization increases C-KIT ${ }^{+}$ICCs in 3-week-old $E d n r^{-/-}$mice. To test the role of TNF- $\alpha$ in enterocolitis, we administered anti-TNF- $\alpha$ neutralizing antibodies in 2 -week-old $E d n r^{-/-}$mice and WT mice. In the proximal and middle colon of 3-week-old Ednrb-/- mice, TNF- $\alpha$ neutralization moderately increased C-KIT ${ }^{+}$ICCs (Supplemental Figure 14A) and C-KIT mRNA levels (Supplemental Figure 14B). CD34 mRNA was expressed at the same levels throughout the colon of $E d n r b^{-/-}$and WT mice (Supplemental Figure 14B).

This suggests that TNF- $\alpha$ may serve as a major mediator in the inflamed colon of HAEC, and that $\mathrm{F} 4 / 80^{+} \mathrm{CD} 11 \mathrm{~b}^{+} \mathrm{CD} 11 \mathrm{c}$ macrophage depletion or TNF- $\alpha$ neutralization may restore the phenotype of ICCs.

TNF- $\alpha$ decreases C-KIT expression and inhibits pacemaker currents in ICCs. We next explored the effects of TNF- $\alpha$ on isolated primary ICCs. Double immunofluorescent staining revealed that TNF- $\alpha$ treatment decreased C-KIT expression but did not affect CD34 expression (Figure 10A). The amplitudes of pacemaker currents in ICCs were significantly decreased by TNF- $\alpha$ treatment (Figure 10, B and C).
$T N F-\alpha$ regulates miR-221 via activation of $N F-\kappa B$. TargetScan software was used to predict possible miRNA molecules targeting C-KIT, revealing that miR-221 was the most likely miRNA targeting C-KIT in both mice and humans (Figure 11A).

TNF- $\alpha$ has long been known to induce NF- $\kappa$ B signaling, typically by translocating the p65 (RelA) DNA-binding factor to the nucleus to initiate the expression of miRNAs (28). In isolated primary ICCs, TNF- $\alpha$ increased miR-221 expression and induced phosphorylation of p65 (p-p65) but inhibited C-KIT expression. Moreover, pyrrolidinedithiocarbamate ammonium (PDTC), an NF- $\kappa B$ inhibitor, was able to inhibit TNF- $\alpha$-induced phosphorylation of $\mathrm{p} 65$, reduced miR-221 expression, and restored C-KIT expression (Figure 11, B-D; and Supplemental Figure 15A). Furthermore, inhibition of miR-221 with an antisense inhibitor in primary ICCs treated with TNF- $\alpha$ recovered the expression of C-KIT but without affecting CD34 expression (Figure 11, E and F; and Supplemental Figure 15B).

These results show that TNF- $\alpha$ inhibits C-KIT expression via miR-221, by activating the NF- $\kappa$ B pathway (Figure $11 G$ ). 
A

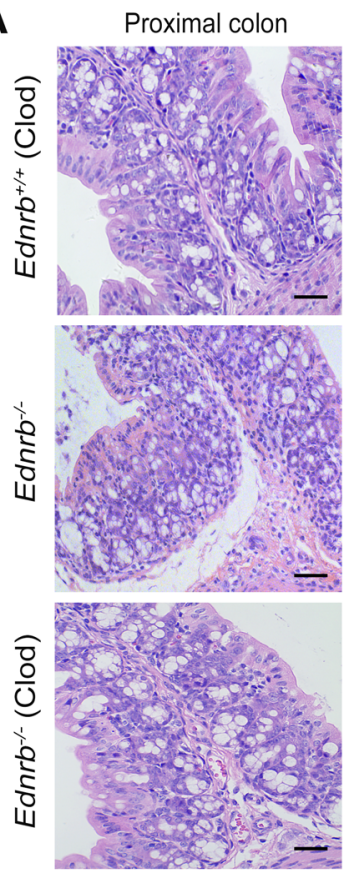

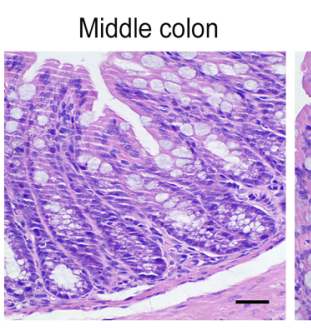
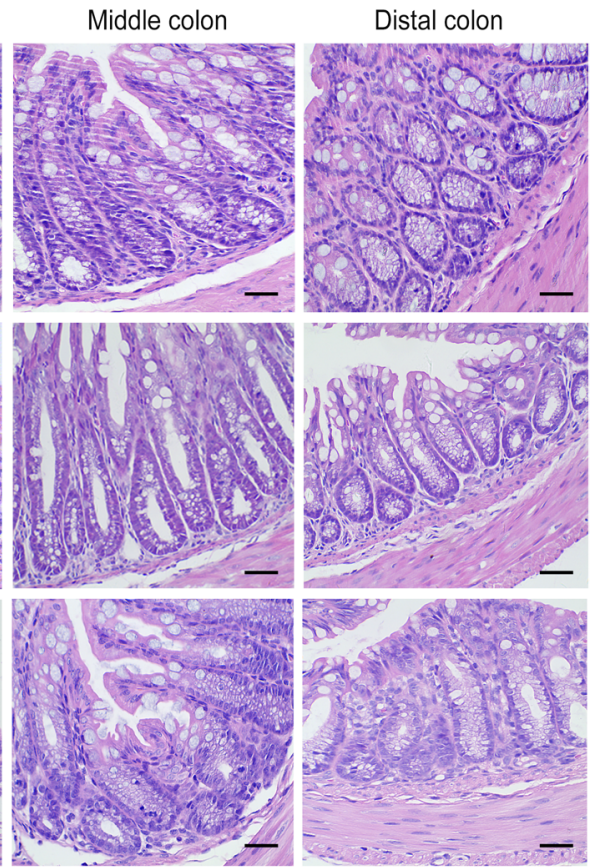
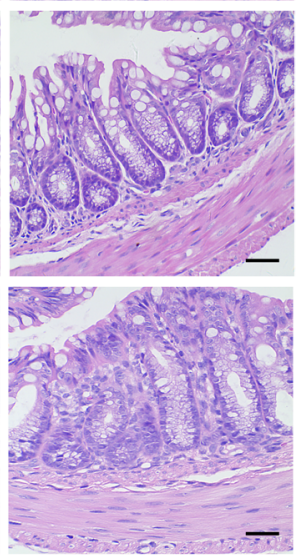

B

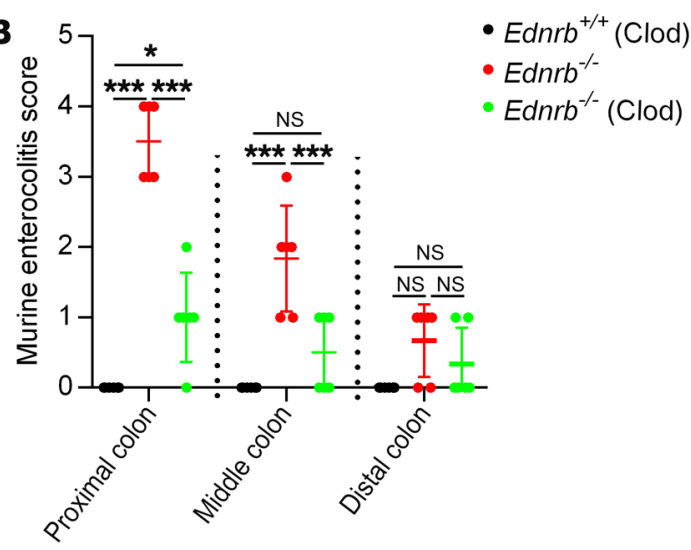

Figure 6. Clod treatment alleviates colitis in 3-week-old Ednrb-/- mice. (A) H\&E staining of proximal, middle, and distal colon from 3-week-old Ednrb/ ${ }^{+/+}$and $E_{n n r b^{-/-}}$mice treated with Clod. Scale bars: $100 \mu \mathrm{m}$. (B) An enterocolitis grading system was used to evaluate inflammation. Data shown represent results from 6 independent experiments. One-way ANOVA: ${ }^{*} P<0.05 ;{ }^{* *} P<0.001$.

\section{Discussion}

In this study, we found that macrophages infiltrated and polarized into proinflammatory macrophages in the proximal dilated colon affected by HAEC in both humans and mice, where they produced a large amount of TNF- $\alpha$ to inhibit C-KIT expression in ICCs. The loss of C-KIT ${ }^{+}$phenotype impaired pacemaker function of ICCs, resulting in persistent colonic dysmotility. Moreover, we report a mechanism by which TNF- $\alpha$ inhibited the C-KIT expression in ICCs via the NF- $\mathrm{kB} / \mathrm{miR}-221$ pathway. These observations increase our knowledge about the etiology of HAEC, and also give a promising therapeutic target for HAEC.

Our findings prove that macrophage-related inflammation directly promotes the development of HAEC in the dilated colon. In the inflamed proximal dilated colon, macrophage infiltration dramatically increased, accompanied by the loss of C-KIT ${ }^{+}$phe- notype in ICCs. Furthermore, macrophages polarized to the $\mathrm{CD} 45^{+} \mathrm{F} 4 / 80^{+} \mathrm{CD} 11 \mathrm{~b}^{+} \mathrm{CD} 11 \mathrm{c}^{-}$proinflammatory phenotype, with massive production of iNOS and TNF- $\alpha$. Previous studies found that macrophage activation was involved in the phenotypic switch of ICCs in trinitrobenzene sulfonic acid-induced ulcerative colitis in rats (29). TNF- $\alpha$ secreted by proinflammatory macrophages can result in KIT loss in ICCs in diabetic mice with gastroparesis (30). These studies illustrate the role of macrophages in phenotypic switches of ICCs in inflamed gastrointestinal tract disease. In the current study, we confirmed the roles of $\mathrm{CD}^{4} 5^{+} \mathrm{F} 4 / 8 \mathrm{O}^{+} \mathrm{CD} 11 \mathrm{~b}^{+} \mathrm{CD} 11 \mathrm{c}^{-}$macrophages and their product TNF- $\alpha$ in regulation of C-KIT expression in ICCs via macrophage depletion or TNF- $\alpha$ neutralization studies. Both studies restored C-KIT expression in ICCs in the proximal dilated colon. It is noteworthy that CD34 expression remained unchanged in $E d n r^{-/-}$mice and WT mice regardless of age and colon segments. Parallel loss of both ANO1 and C-KIT was confirmed in the proximal dilated colon of 3-week-old Ednrb ${ }^{-/}$mice, suggesting a phenotypic switch of the ICCs during HAEC.

The loss of C-KIT ${ }^{+}$phenotype of ICCs was also found in the distal aganglionic colon in the absence of inflammatory cell infiltration. The loss of the ICC phenotype may be due to the lack of SCF, which maintains C-KIT expression, survival, and functional activity of ICCs, in the aganglionic colon (31). This is different from the absence of C-KIT ${ }^{+}$ ICCs in the inflamed proximal colon.

HSCR is characterized by aganglionosis of the distal colon. Previous studies confirmed that an immature enteric nervous system (ENS) plays a significant role in necrotizing enterocolitis (NEC), another common intestinal inflammatory disease in neonates. The intestinal dysmotility noted in NEC patients was thought to be caused by an immature ENS $(32,33)$. Moreover, examination of the myenteric plexus and external submucosal plexus of NEC patients revealed a noticeable reduction in glial cells concomitant with the gradual deterioration of nerve cells, both findings predominating in the antimesenteric intestinal circumference, where ischemic lesions tended to appear first $(34,35)$. However, immaturity or ischemia is not commonly found in HAEC. The clinical observation that HAEC is not alleviated by removal of the aganglionic colon also suggests that ENS insufficiency of the distal colon in HSCR plays a less important role in HAEC (36).

MiRNAs have been reported to play important roles in regulating C-KIT expression. MiR-494 downregulates C-KIT and inhibits gastrointestinal stromal tumor cell proliferation (37). We used bioinformatics analysis to speculate possible miRNAs targeting C-KIT, and found that miR-221 may be involved in regulation of C-KIT expression. MiR-221 has a variety of effects by specifically binding to the 3 '-UTR of C-KIT mRNA in eukaryocytes $(38,39)$. In isolated ICCs, we found that TNF- $\alpha$ induced miR-221 expression, and inhibited C-KIT expression. Moreover, an NF- $\kappa$ B inhibitor inhibited phosphorylation of $\mathrm{p} 65$ and miR-221 expression, accompanied by increased expression of C-KIT. The miR-221 antisense inhibitor also reversed 
A
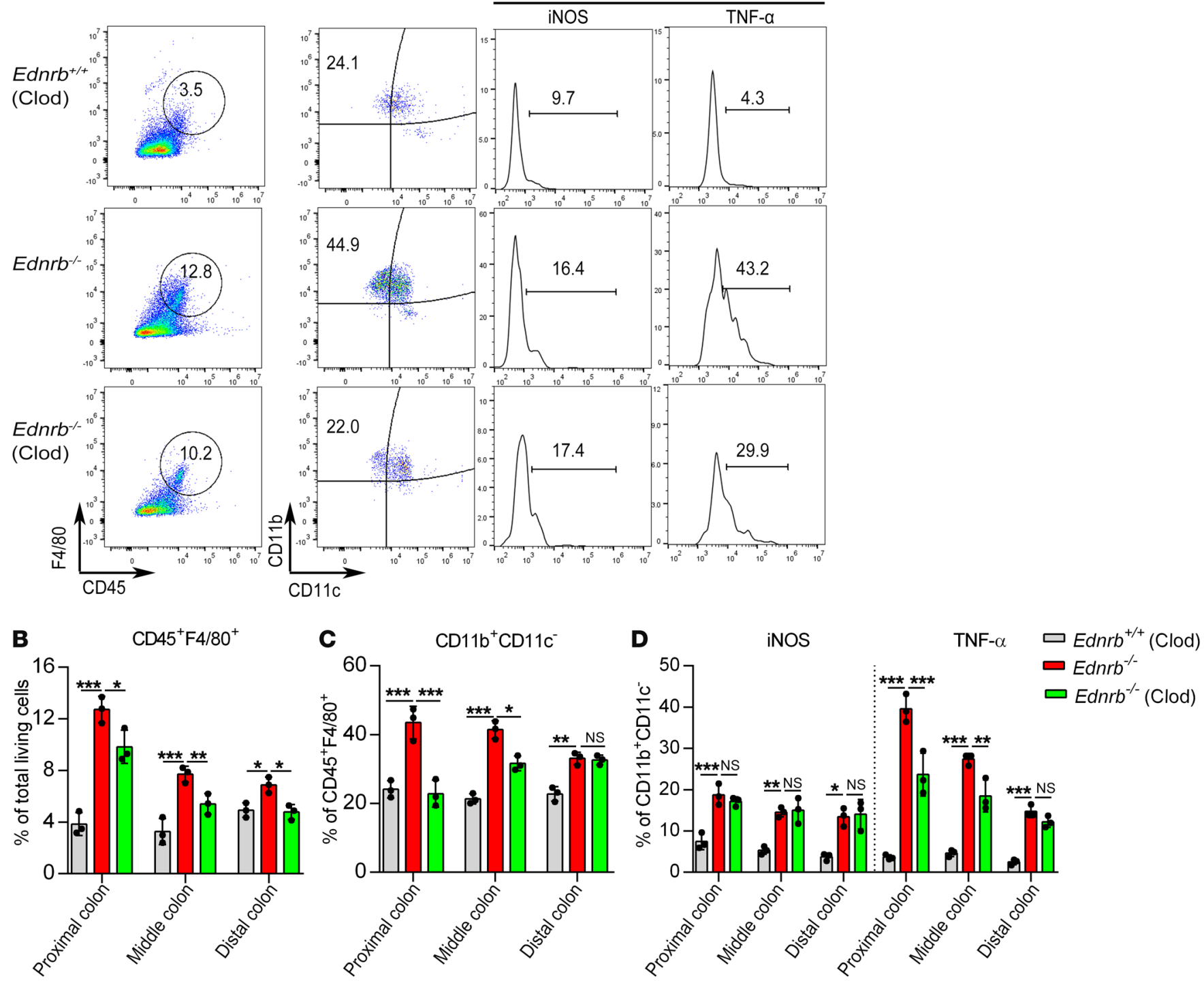

Figure 7. Proinflammatory M1 macrophages decrease in the proximal colon of 3-week-old Ednrb-/- mice after Clod treatment. (A) Representa-

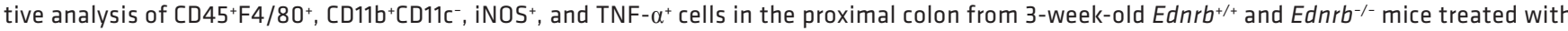
Clod. (B) Percentage of $C D 45^{+} \mathrm{F} 4 / 80^{+}$cells among total viable cells in colonic tissue. (C) Percentage of CD11 b $\mathrm{C}^{+} \mathrm{CD} 11 \mathrm{c}^{-}$cells among $\mathrm{CD} 45^{+} \mathrm{F} 4 / 80^{+}$cells. (D) Percentage of iNOS ${ }^{+}$cells and TNF- $\alpha^{+}$cells among CD11b+CD11c ${ }^{-}$cells. Data shown represent results from 3 independent experiments. One-way ANOVA: ${ }^{*} P<0.05 ;{ }^{* *} P<0.01 ;{ }^{* *} P<0.001$

TNF- $\alpha$-induced suppression of C-KIT. These findings indicate the role of the TNF- $\alpha / \mathrm{NF}-\kappa \mathrm{B} / \mathrm{miR}-221$ pathway in suppressing C-KIT expression.

Phenotypic changes of ICCs resulted in abnormal amplitudes and rhythms of colonic slow waves from the proximal to distal colon in 3-week-old Ednrb/-- mice. Since colonic slow waves are formed by propagating proximal colon peristalsis toward the distal colon, phenotypic changes of ICCs may be responsible for persistent intestinal dysfunction in HSCR patients after a pullthrough procedure, leading to persistent problems with constipation or fecal incontinence. Based on these observations, it is necessary to remove an adequate length of dilated colon to avoid the reoccurrence of HAEC and improve intestinal motility in children undergoing surgery.
Previous studies found a variety of cytokines, including IL-1, IL-6, IL-8, and IFN- $\gamma$, engaged in the phenotypic switch of ICCs in the inflamed intestine, including inflammatory bowel disease and $\operatorname{HAEC}(3,40,41)$. In this study, we found that TNF- $\alpha$ induced the expression of miR-221 in an NF- $\kappa B-d e p e n d e n t$ manner in ICCs. It is possible that there are other NF- $\mathrm{BB}-$ dependent proinflammatory cytokines able to induce the expression of miR-221, though few studies regarding this issue have been published. Future research will use a cytokine array or genome-wide screens of miRNAs to explore the possible cytokines or miRNAs and their interaction in HAEC.

In conclusion, we found that enterocolitis mostly affects the proximal dilated colon in HAEC, where $\mathrm{CD} 45^{+} \mathrm{F} 4 / 80^{+} \mathrm{CD} 11 \mathrm{~b}^{+}$ $\mathrm{CD} 11 \mathrm{c}^{-}$proinflammatory macrophages produce TNF- $\alpha$. In ICCs, 
A

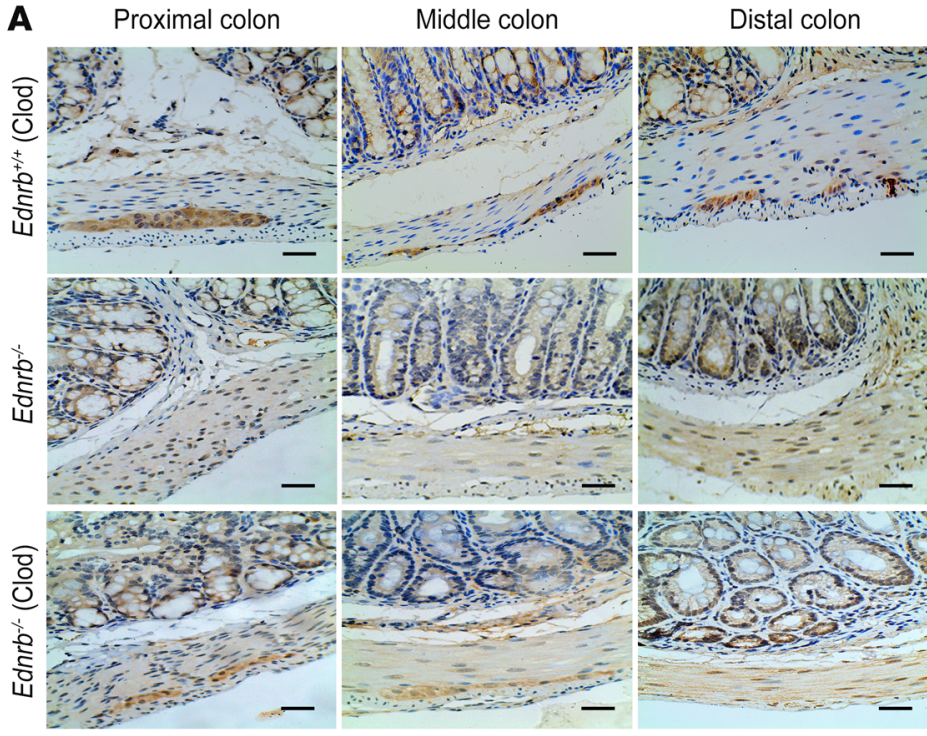

\section{B}

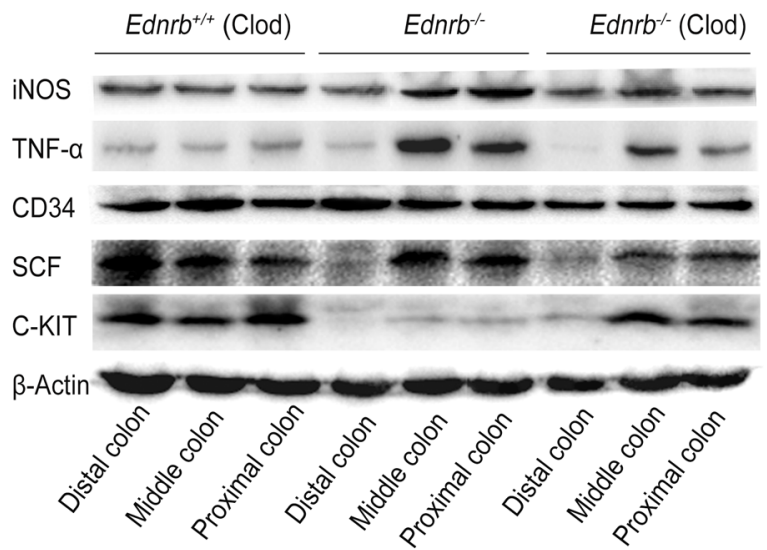

SCF

C-KIT

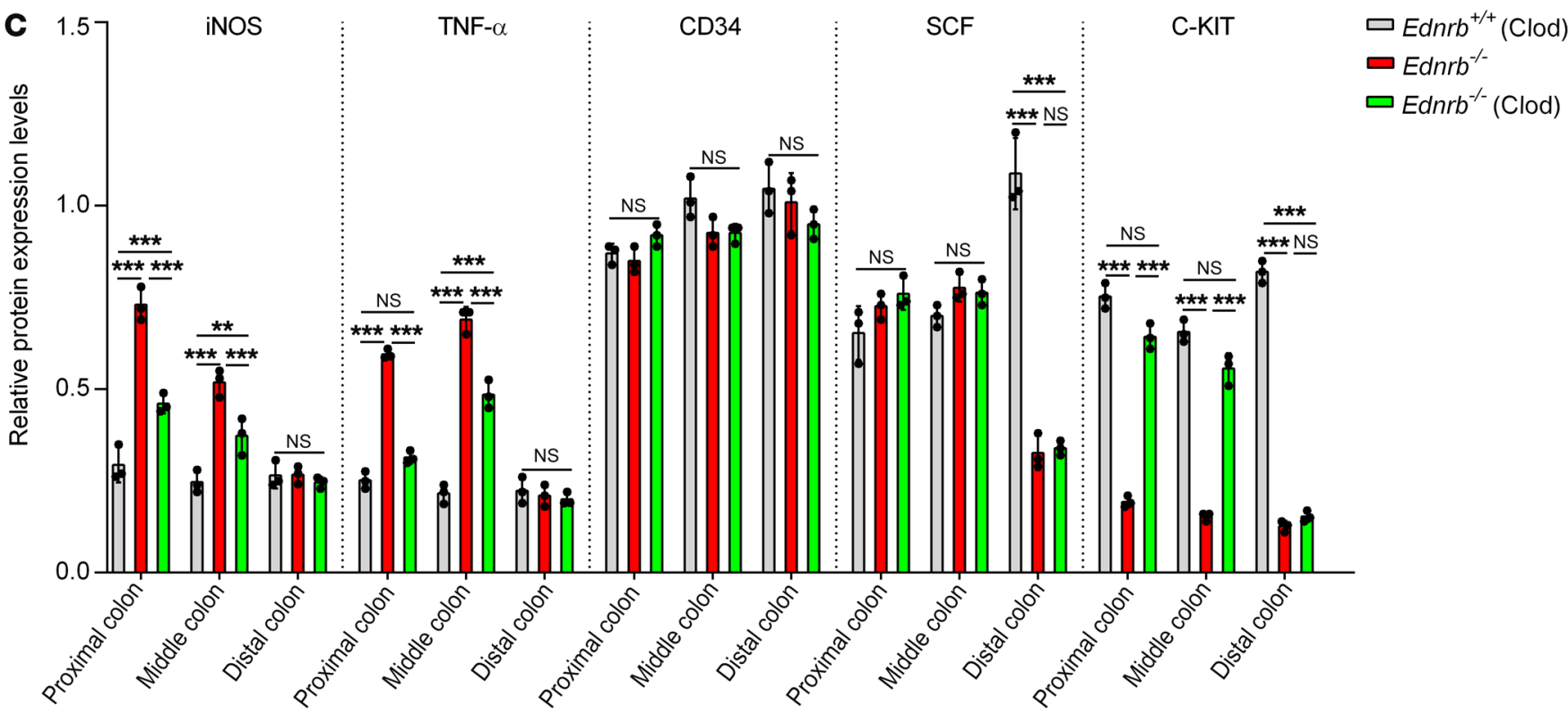

Figure 8. Clod treatment suppresses inflammatory cytokine production and promotes recovery of ICC phenotype in 3-week-old Ednrb-/- mice. (A) Immunohistochemistry staining for C-KIT in ICCs from 3-week-old Ednrb ${ }^{+/+}$and Ednrb-/- mice treated with Clod. Scale bars: $100 \mu \mathrm{m}$. (B) Western blot analysis of colon from 3-week-old Ednrb ${ }^{+/+}$and Ednrb ${ }^{-/-}$mice treated with Clod. (C) Semiquantitative analysis of protein expression levels, with each protein being normalized to $\beta$-actin. Data shown represent results from 3 independent experiments. One-way ANOVA: ${ }^{* *} P<0.01 ;{ }^{* *} P<0.001$.

TNF- $\alpha$ activates NF- $\kappa$ B to upregulate the expression of miR-221, leading to the suppression of C-KIT expression and abnormal intestinal peristalsis. Intestinal obstruction secondary to abnormal intestinal peristalsis can lead to an increased proinflammatory environment due to fecal retention and bacterial overgrowth. Therefore, a vicious cycle can occur, further promoting the development of HAEC. Treatment targeting macrophages or TNF- $\alpha$ may represent promising therapeutics for HAEC in the future.

\section{Methods}

Further details are provided in Supplemental Methods.

Human tissue specimen collection. Between January 2014 and December 2016, specimens of resected colon were collected from
96 patients during pull-through procedures at our institution. All of them were pathologically diagnosed with HSCR. According to the morphology of the colon, specimens were divided into 4 groups: distal narrowed colon, transition zone, dilated colon, and proximal normal colon. Specimens were used for morphological experiments and protein and mRNA studies. The severity of enterocolitis was scored using an established histopathologic scale (17). Based on inflammation severity by histopathologic findings, samples were divided into an HSCR group and an HAEC group.

Murine model of HSCR. A breeding colony of Ednr $b^{\text {tmIYwa/J }}$ heterozygous mice ( $E d n r b^{\text {tmlYwa/J }}$ on a hybrid C57BL/6J-129Sv background) was purchased from The Jackson Laboratory (JAX-003295). A PCRbased assay was used to identify the mouse genotype. The heterozy- 
A

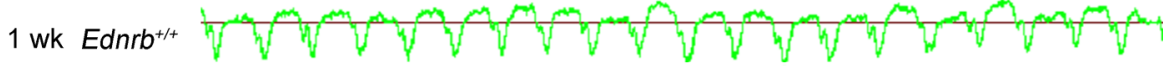

1 wk Ednrb-1-

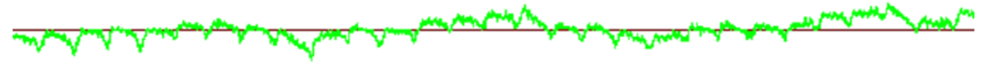

$2 \mathrm{wk} E d n \mathrm{~b}^{+/+} \mathrm{y}$ W

2 wk Ednrb-

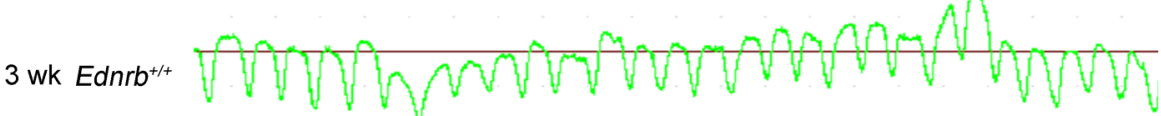

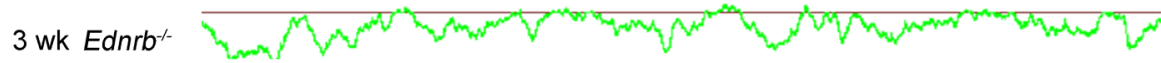

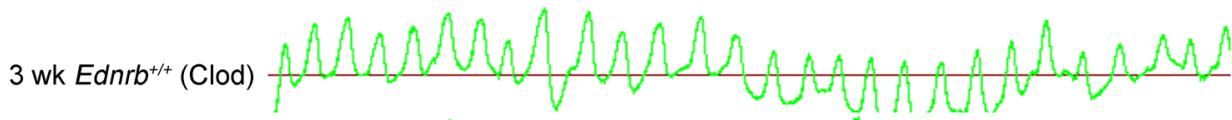

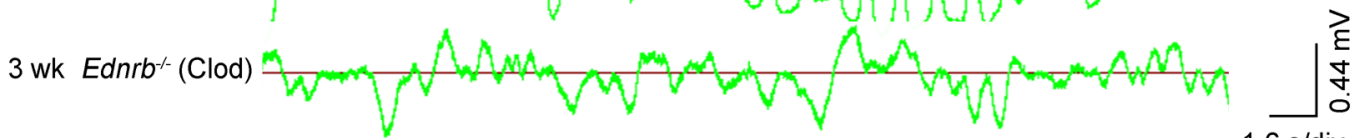

$1.6 \mathrm{~s} / \mathrm{div}$

B

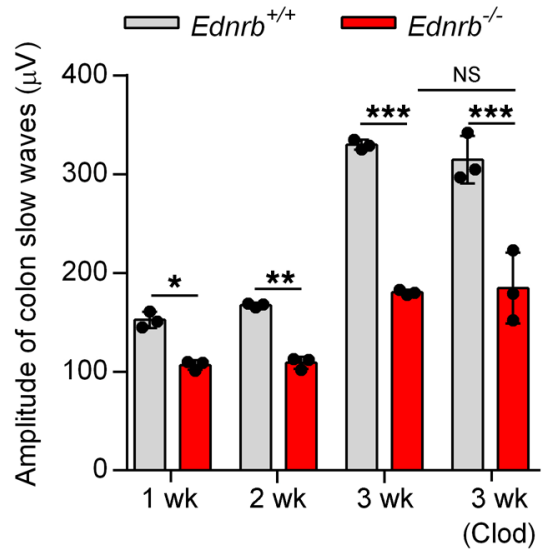

C

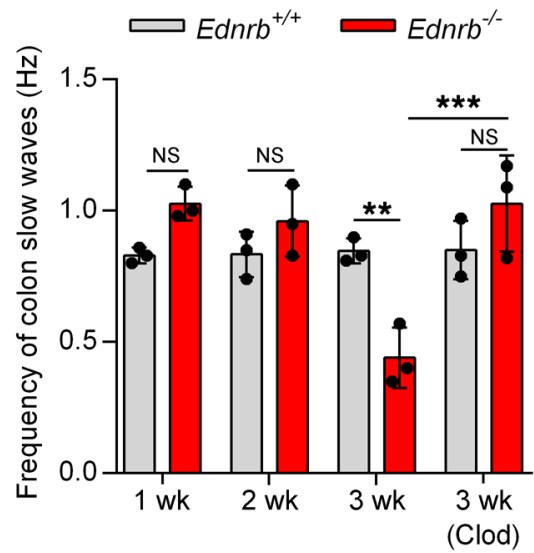

Figure 9. Clod treatment restores murine colon slow waves. (A) Colonic slow waves from 1-, 2-, and 3-week-old Ednrb ${ }^{+/+}$and $E d n r b^{-/-}$mice, and

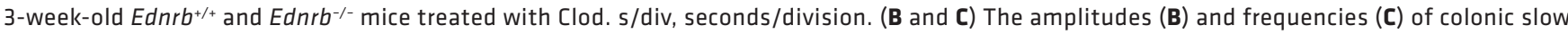
waves of 1-, 2-, and 3-week-old Ednrb ${ }^{+/+}$and $E d n r b^{-/-}$mice are shown. Data shown represent results from 3 independent experiments. One-way ANOVA: ${ }^{*} P<0.05 ;{ }^{* *} P<0.01 ;{ }^{* *} P<0.001$.

gote littermates $\left(E d n r b^{+/}\right)$were used as the breeder mice, while the WT $\left(E d n r b^{+/+}\right)$and homozygous $\left(E d n r b^{-/-}\right)$mice were used for experiments. The $E d n r b^{+/+}$and $E d n r b^{-/-}$mice were sacrificed at the end of the first, second, and third weeks after birth and their colons harvested. A murine enterocolitis grading system was used to evaluate the severity of enterocolitis (42).

Colon histopathology and IHC. Since the dilated colon of 3-weekold $E d n r b^{-/-}$mice extended to nearly the proximal colon, we divided mouse colon into distal colon, middle colon, and proximal colon, which was analogous to the human distal narrowed colon, transition zone, and dilated colon. The expression of C-KIT in human and mouse colonic tissues was studied by IHC staining.

Immunofluorescence staining. Immunofluorescence (IF) staining of the paraffin-embedded colon sections was performed. Antibodies used in IF are listed in Supplemental Table 1.

Whole-mount colon specimens were prepared, and incubated with primary antibodies (Supplemental Table 1) at $4^{\circ} \mathrm{C}$ overnight.
Specimens were washed and then stained with goat anti-rabbit IgG Cy3-conjugated secondary antibody (catalog WGZ5-715-165-150, Servicebio) or goat anti-rat IgG FITC-conjugated secondary antibody (catalog GB22403, Servicebio) for 1 hour at room temperature. The specimens were examined using confocal microscopy.

Flow cytometry. Intestinal leukocytes of the 1-, 2-, and 3-week-old $E d n r b^{-/-}$and $E d n r b^{+/+}$mice, along with the 3-week-old mice treated with Clod, were isolated as described previously (43). Single-cell suspensions were incubated with combinations of antibodies (Supplemental Table 2). Cells were analyzed on a FACSCalibur (BD Immunocytometry Systems, BD Biosciences). Analysis was performed using FlowJo software (version 10, FlowJo LLC).

Quantitative real-time PCR. Quantitative real-time PCR was performed as previously described (11). PCR primers used in the study are listed in Supplemental Table 3 as human samples, in Supplemental Table 4 as murine samples, and in Supplemental Table 5 as miRNA detection. 
A

CD34 C-KIT

DAPI Merge
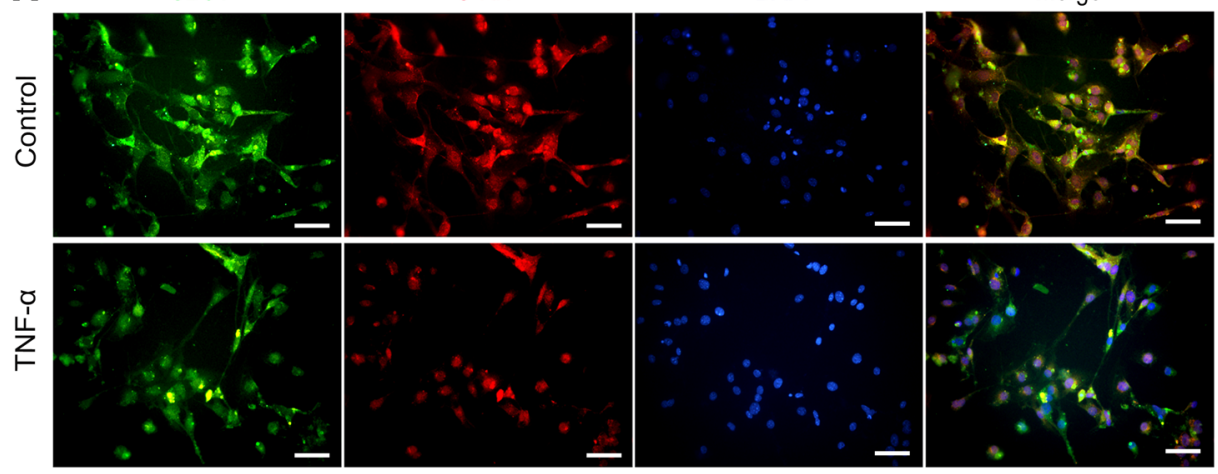

B

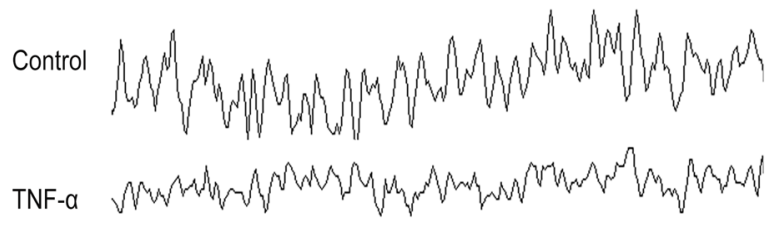

C

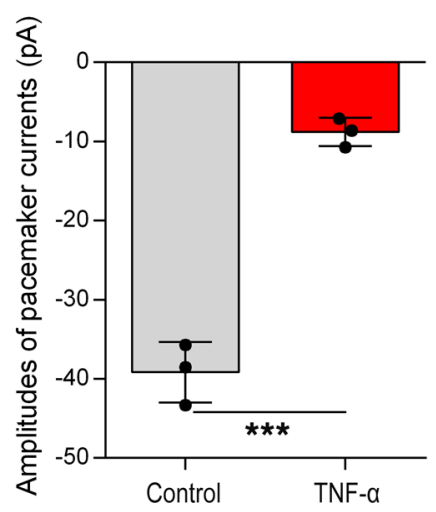

Figure 10. TNF- $\alpha$ induces downregulation of C-KIT and inhibits the pacemaker currents of isolated ICCs. (A) C-KIT and CD34 immunofluorescence double staining was used to identify the ICC phenotype with or without TNF- $\alpha$ treatment. Green, CD34; red, C-KIT; blue, DAPI. Scale bars: $100 \mu \mathrm{m}$. (B) Pacemaker currents of ICCs were recorded using the whole-cell mode of the patch clamp technique. (C) Amplitudes of pacemaker currents with or without TNF- $\alpha$ treatment. Data shown represent results from 3 independent experiments. Student's $t$ test: ${ }^{* * *} P<0.001$.

Western blot. Western blotting analysis was performed as previously described (11). The antibodies used are listed in Supplemental Table 6. Protein bands were quantified by densitometry with Quantity One Software (Bio-Rad).

Measurement of colonic electrical activity. Mice were anesthetized with an i.p. injection of $80 \mathrm{mg} / \mathrm{kg}$ of $1 \%$ sodium pentobarbital (catalog 1507002, MilliporeSigma). After opening of the abdominal cavity, 2 bipolar electrodes were placed on the serosa circumferentially around the lumen at an interval of $2 \mathrm{~cm}$. Mouse colon myoelectrical activity was recorded and analyzed using a multiple-channel recorder (model BL-420E+xz, Chengdu Techman Software Co. Ltd.). The amplifier was set at a cutoff frequency of $52 \mathrm{~Hz}$. Tracings were displayed on an online monitor and saved on a hard disk with a sampling frequency of $100 \mathrm{~Hz}$.

Macrophage depletion by i.p. injection of Clod in vivo. Two-weekold mice were treated with $100 \mu \mathrm{L}$ of Clod $(5 \mathrm{mg} / \mathrm{mL}$ ) (catalog F70101C-N, FormuMax Scientific Inc.) via i.p. injection at 1 day and 4 days before measurement of endpoints. Mice were sacrificed at the end of the third week after birth to harvest colons.

TNF- $\alpha$ neutralization in vivo. To neutralize TNF- $\alpha$ in vivo, 2-weekold mice were treated with $300 \mu \mathrm{g}$ of anti-TNF- $\alpha$ monoclonal antibody (clone XT3.11, catalog BE0058, Bio X Cell) via i.p. injection at 1 and 4 days before measurement of endpoints. Mice were sacrificed at the end of the third week after birth, and colons were harvested for IHC staining and quantitative real-time PCR.

ICC isolation and culture. Primary ICCs were isolated from the colon of 3-week-old $E d n r b^{+/+}$mice as we have previously described
(11). ICCs were cultured at $37^{\circ} \mathrm{C}$ in a $5 \% \mathrm{CO}_{2}$ incubator. After 24 hours of incubation, cells in the experimental group were incubated with 5 $\mathrm{ng} / \mathrm{mL} \mathrm{TNF}-\alpha$ (catalog 654245, MilliporeSigma). One day later, cells were collected and used for IF staining, protein and mRNA assay, miRNA detection, and recording of pacemaker currents.

For treatment groups, primary ICCs were cultured for 24 hours, and then $20 \mu \mathrm{M}$ PDTC (catalog S1808, Beyotime) or $100 \mathrm{nM}$ micrOFF mmu-miR-221-3p inhibitor (catalog miR30000669-4-5, Ribobio) was added to the cell culture medium. One hour later, cells in the experimental group were incubated with $5 \mathrm{ng} / \mathrm{mL}$ TNF- $\alpha$. One day later, cells were harvested for protein, mRNA, and miRNA assays.

Patch clamp studies of ICCs. A conventional dialyzed whole-cell patch clamp configuration was used to record membrane currents (voltage clamp) and potentials (current clamp, $\mathrm{i}=0$ ) from cells. Membrane currents or transmembrane potentials were amplified with an Axopatch 200B patch clamp amplifier (Molecular Devices) and digitized with a 16-bit analog-to-digital converter (Digidata 1322A, Molecular Devices). The currents and potentials were stored directly online using pCLAMP software (version 9.2, Molecular Devices). Data were sampled at $4 \mathrm{kHz}$ and filtered at $2 \mathrm{kHz}$ for whole-cell experiments. Mini-Digi with Axoscope (version 9.2, Molecular Devices) was used to monitor changes in holding currents (basal currents) throughout each experiment. All data were analyzed using Clampfit (version 9.2, Molecular Devices) and GraphPad Prism software (version 6.0, GraphPad Software). The pipette tip resistance ranged between 3 and $6 \mathrm{M} \Omega$ for whole-cell 
A

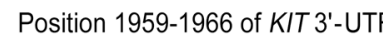

hsa-miR-221-3p

Position $1853-1860$ of KIT 3'-UTR

mmu-miR-221-3p

B

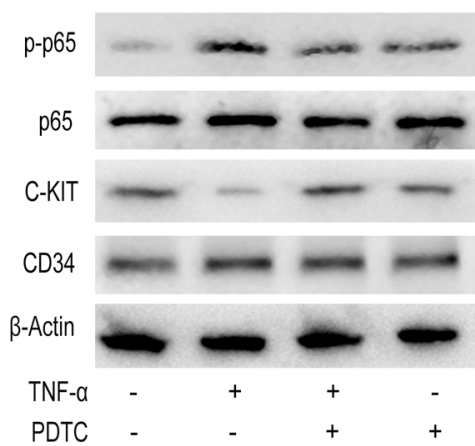

$\mathbf{E}$

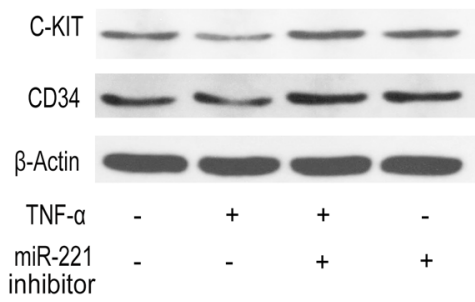

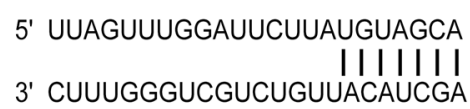

5' UUUGUAAAUAUUGAAAUGUAGCA
3' CUUUGgGUCGUCUGUUACAUCGA

C

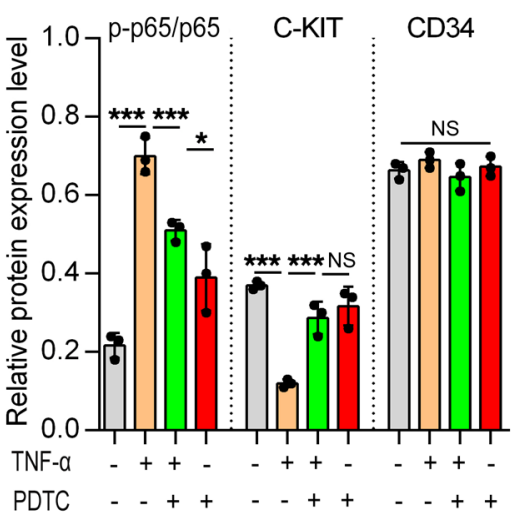

$\mathbf{F}$

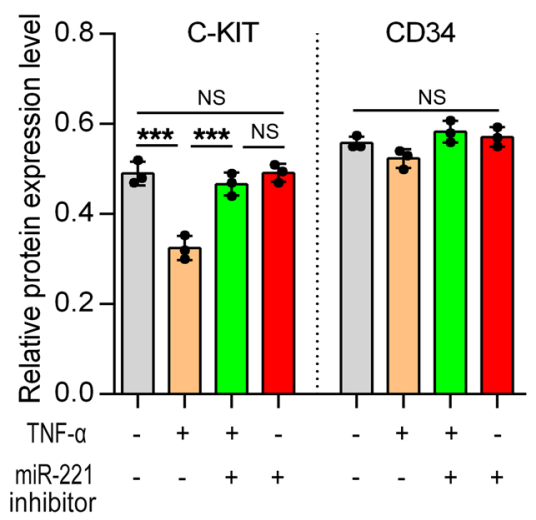

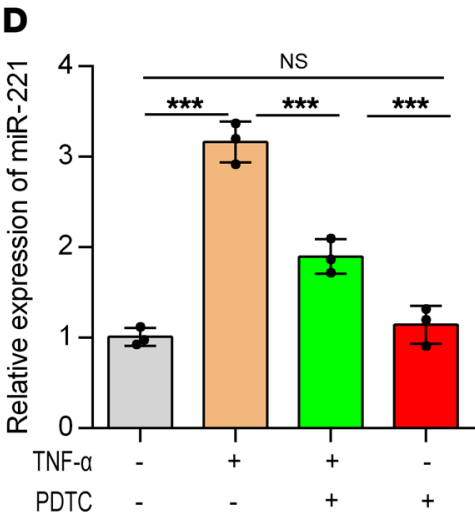

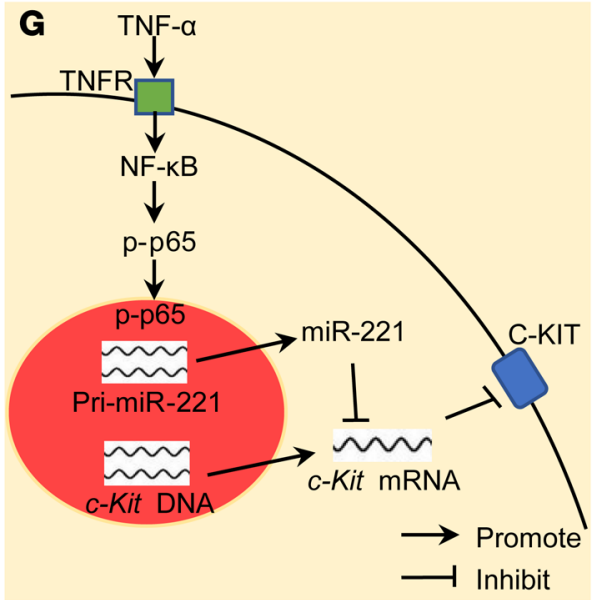

Figure 11. The TNF- $\alpha /$ NF-KB/miR-221 signaling pathway is involved in C-KIT expression in isolated ICCs. (A) TargetScan software was used to predict the possible miRNAs that could target the $3^{\prime}$-UTR of $c$-Kit mRNA in humans and mice. (B) Western blotting of isolated ICCs with or without TNF- $\alpha$ and PDTC treatment. (C) Semiquantitative analysis of protein expression levels with each protein normalized to $\beta$-actin. (D) MiR-221 expression levels with or without TNF- $\alpha$ and PDTC treatment. (E and F) Western blot analysis of C-KIT and CD34 in isolated ICCs with or without TNF- $\alpha$ and miR-221 inhibitor treatment. Semiquantitative analysis of protein expression levels with each protein normalized to $\beta$-actin. Data shown represent results from 3 independent experiments. (G) The signaling pathway involving TNF- $\alpha$, miR-221, and C-KIT in ICCs. One-way ANOVA: ${ }^{*} P<0.05 ;{ }^{* * *} P<0.001$.

recordings, and experiments on ICCs were conducted at $30^{\circ} \mathrm{C}$ with the use of a Thermoclamp-1 temperature control system (AutoMate Scientific).

Immunofluorescence staining of ICCs. IF staining of the cells was performed. Primary antibodies were anti-C-KIT (1:200 dilution; catalog ab171227, Abcam) or anti-CD34 (1:200 dilution; catalog EP373Y, Abcam) antibodies. Secondary antibodies were goat anti-rat IgG Cy3-conjugated (catalog GB21302, Servicebio) or goat anti-rabbit IgG FITC-conjugated (catalog GB22403, Servicebio) secondary antibodies. Cells were visualized under a laser scanning confocal microscope.

Statistics. All images were analyzed using Image-Pro Plus (Media Cybernetics Corp.) and merged using Photoshop CS5 software. Data were analyzed using the GraphPad Prism software package (version 6) and results presented as the mean \pm SEM. Differences between 2 groups were analyzed using an unpaired 2-tailed Student's $t$ test with Welch's correction. ANOVA with Tukey's correction was used to investigate more than 2 groups. $P$ values less than 0.05 were considered statistically significant.

Study approval. All protocols were approved by the Ethics Review Committee of Tongji Hospital. All study procedures complied with regulations in the Declaration of Helsinki, and all guardians provided their written informed consent. The protocols for animal studies were approved by the Institutional Animal Care and Use Committee at Tongji Hospital (permit 20160221). 


\section{Author contributions}

JF and GEB conceived and designed research. XC, XM, CF, CJ, and JW performed experiments and analyzed data. ZL, KF, XX, $\mathrm{LY}$, and XW collected human tissue samples. XC, XM, JY, and BW interpreted results of experiments and prepared figures. $\mathrm{XC}, \mathrm{HZ}$, JF, NL, and KMA drafted the manuscript. GEB edited and revised the manuscript. JF approved the final version of manuscript.

\section{Acknowledgments}

We thank Yuan Yu, Xianguang Lin, and Xinwu Hu for their technical support with the confocal microscopy, patch clamp technique, and the electrophysiological recorder. We also thank John Sherman for help in revising the manuscript. This study was supported by grants from the National Natural Science Foundation of China (81270441 and 81200266), the National Key Research and Development Project (2016YFE0203900), and the Sanming Project of Medicine in Shenzhen (SZSM201812055).

Address correspondence to: Gail E. Besner, Department of Pediatric Surgery, Center for Perinatal Research, Nationwide Children's Hospital, 700 Children's Drive, Columbus, Ohio 43205, USA. Phone: 614.722.3914; Email: gail.besner@nationwidechildrens. org. Or to: Jiexiong Feng, Department of Pediatric Surgery, Tongji Hospital, Tongji Medical College, Huazhong University of Science and Technology, 1095 Jiefang Avenue, Wuhan 430030, China. Phone: 86.027.83665289; Email: fengjiexiong@126.com.
1. Langer JC. Hirschsprung disease. Curr Opin Pediatr. 2013;25(3):368-374.

2. Demehri FR, Halaweish IF, Coran AG, Teitelbaum DH. Hirschsprung-associated enterocolitis: pathogenesis, treatment and prevention. Pediatr Surg Int. 2013;29(9):873-881.

3. Jiao CL, Chen XY, Feng JX. Novel insights into the pathogenesis of Hirschsprung's-associated enterocolitis. Chin Med J. 2016;129(12):1491-1497.

4. Sanders KM. Spontaneous electrical activity and rhythmicity in gastrointestinal smooth muscles. Adv Exp Med Biol. 2019;1124:3-46.

5. Yamataka A, et al. A lack of intestinal pacemaker (c-kit) in aganglionic bowel of patients with Hirschsprung's disease. J Pediatr Surg. 1995;30(3):441-444.

6. Rolle U, Piotrowska AP, Nemeth L, Puri P. Altered distribution of interstitial cells of Cajal in Hirschsprung disease. Arch Pathol Lab Med. 2002;126(8):928-933.

7. Gfroerer S, Rolle U. Interstitial cells of Cajal in the normal human gut and in Hirschsprung disease. Pediatr Surg Int. 2013;29(9):889-897.

8. Horisawa M, Watanabe Y, Torihashi S. Distribution of c-Kit immunopositive cells in normal human colon and in Hirschsprung's disease. J Pediatr Surg. 1998;33(8):1209-1214.

9. Sanders KM, Koh SD, Ward SM. Interstitial cells of cajal as pacemakers in the gastrointestinal tract. Annu Rev Physiol. 2006;68:307-343.

10. Adachi Y, et al. Phenotypic alteration of interstitial cells of Cajal in idiopathic sigmoid megacolon. J Gastroenterol. 2008;43(8):626-631.

11. Wei J, et al. Effects of lipopolysaccharide-induced inflammation on the interstitial cells of Cajal. Cell Tissue Res. 2014;356(1):29-37.

12. Bain CC, Mowat AM. Intestinal macrophages specialised adaptation to a unique environment. Eur J Immunol. 2011;41(9):2494-2498.

13. Lissner D, et al. Monocyte and M1 macrophage-induced barrier defect contributes to chronic intestinal inflammation in IBD. Inflamm Bowel Dis. 2015;21(6):1297-1305.

14. Wei J, Besner GE. M1 to M2 macrophage polarization in heparin-binding epidermal growth factor-like growth factor therapy for necrotizing enterocolitis. J Surg Res. 2015;197(1):126-138.

15. Kaji N, et al. Nitric oxide-induced oxidative stress impairs pacemaker function of murine interstitial cells of Cajal during inflammation. Pharmacol Res. 2016;111:838-848.
16. Eisenman ST, Gibbons SJ, Verhulst PJ, Cipriani G, Saur D, Farrugia G. Tumor necrosis factor alpha derived from classically activated "M1" macrophages reduces interstitial cell of Cajal numbers. Neurogastroenterol Motil. 2017;29(4):10.1111/nmo.12984.

17. Elhalaby EA, Teitelbaum DH, Coran AG, Heidelberger KP. Enterocolitis associated with Hirschsprung's disease: a clinical histopathological correlative study. J Pediatr Surg. 1995;30(7):1023-1026.

18. Hosoda K, et al. Targeted and natural (piebald-lethal) mutations of endothelin-B receptor gene produce megacolon associated with spotted coat color in mice. Cell. 1994;79(7):1267-1276.

19. Zhao L, et al. Murine model of Hirschsprung-associated enterocolitis. II: Surgical correction of aganglionosis does not eliminate enterocolitis. JPediatr Surg. 2010;45(1):206-211.

20. Jiang M, et al. Calretinin, S100 and protein gene product 9.5 immunostaining of rectal suction biopsies in the diagnosis of Hirschsprung' disease. Am J Transl Res. 2016;8(7):3159-3168.

21. Yang S, Donner LR. Detection of ganglion cells in the colonic plexuses by immunostaining for neuron-specific marker NeuN: an aid for the diagnosis of Hirschsprung disease. Appl Immunohistochem Mol Morphol. 2002;10(3):218-220.

22. Radenkovic G, Radenkovic D, Velickov A. Development of interstitial cells of Cajal in the human digestive tract as the result of reciprocal induction of mesenchymal and neural crest cells. J Cell Mol Med. 2018;22(2):778-785.

23. Gomez-Pinilla PJ, et al. Ano1 is a selective marker of interstitial cells of Cajal in the human and mouse gastrointestinal tract. Am J Physiol Gastrointest Liver Physiol. 2009;296(6):G1370-G1381.

24. Denning TL, et al. Functional specializations of intestinal dendritic cell and macrophage subsets that control Th17 and regulatory $\mathrm{T}$ cell responses are dependent on the T cell/APC ratio, source of mouse strain, and regional localization. J Immunol. 2011;187(2):733-747.

25. Kawano Y, et al. Colonic pro-inflammatory macrophages cause insulin resistance in an intestinal Ccl2/Ccr2-dependent manner. Cell Metab. 2016;24(2):295-310.

26. Weisser SB, van Rooijen N, Sly LM. Depletion and reconstitution of macrophages in mice. J Vis Exp. 2012;(66):4105.
27. Klein S, et al. Interstitial cells of Cajal integrate excitatory and inhibitory neurotransmission with intestinal slow-wave activity. Nat Commun 2013;4:1630.

28. Boldin MP, Baltimore D. MicroRNAs, new effectors and regulators of NF- $\mathrm{BB}$. Immunol Rev. 2012;246(1):205-220.

29. Kinoshita K, et al. Possible involvement of muscularis resident macrophages in impairment of interstitial cells of Cajal and myenteric nerve systems in rat models of TNBS-induced colitis. Histochem Cell Biol. 2007;127(1):41-53.

30. Neshatian L, Gibbons SJ, Farrugia G. Macrophages in diabetic gastroparesis-the missing link? Neurogastroenterol Motil. 2015;27(1):7-18

31. Tan YY, Ji ZL, Zhao G, Jiang JR, Wang D, Wang JM. Decreased SCF/c-kit signaling pathway contributes to loss of interstitial cells of Cajal in gallstone disease. Int JClin Exp Med. 2014;7(11):4099-4106.

32. Zhou Y, et al. Enteric nervous system abnormalities are present in human necrotizing enterocolitis: potential neurotransplantation therapy. Stem Cell Res Ther. 2013;4(6):157.

33. Zhou Y, Wang Y, Olson J, Yang J, Besner GE. Heparin-binding EGF-like growth factor promotes neuronal nitric oxide synthase expression and protects the enteric nervous system after necrotizing enterocolitis. Pediatr Res. 2017;82(3):490-500.

34. Bush TG. Enteric glial cells. An upstream target for induction of necrotizing enterocolitis and Crohn's disease? Bioessays. 2002;24(2):130-140.

35. Sigge W, Wedel T, Kühnel W, Krammer HJ. Morphologic alterations of the enteric nervous system and deficiency of non-adrenergic non-cholinergic inhibitory innervation in neonatal necrotizing enterocolitis. Eur J Pediatr Surg. 1998;8(2):87-94.

36. Nakamura H, Lim T, Puri P. Inflammatory bowel disease in patients with Hirschsprung's disease: a systematic review and meta-analysis. Pediatr Surg Int. 2018;34(2):149-154.

37. Kim WK, et al. MicroRNA-494 downregulates KIT and inhibits gastrointestinal stromal tumor cell proliferation. Clin Cancer Res. 2011;17(24):7584-7594.

38. Liu X, Cheng Y, Yang J, Xu L, Zhang C. Cell-specific effects of miR-221/222 in vessels: molecular mechanism and therapeutic application. JMol Cell Cardiol. 2012;52(1):245-255. 
39. Felli N, et al. MicroRNAs 221 and 222 inhibit normal erythropoiesis and erythroleukemic cell growth via kit receptor down-modulation. Proc Natl Acad Sci U S A. 2005;102(50):18081-18086.

40. Imamura A, Puri P, O'Briain DS, Reen DJ. Mucosal immune defence mechanisms in enterocolitis complicating Hirschsprung's disease. Gut.
1992;33(6):801-806.

41. Mikkelsen HB. Interstitial cells of Cajal, macrophages and mast cells in the gut musculature: morphology, distribution, spatial and possible functional interactions. J Cell Mol Med. 2010;14(4):818-832.

42. Cheng Z, et al. Murine model of Hirschsprung-as- sociated enterocolitis. I: phenotypic characterization with development of a histopathologic grading system. J Pediatr Surg. 2010;45(3):475-482.

43. Zigmond $\mathrm{E}$, et al. Ly6C hi monocytes in the inflamed colon give rise to proinflammatory effector cells and migratory antigen-presenting cells. Immunity. 2012;37(6):1076-1090. 